ARTICLE

Received 18 Feb 2015 | Accepted 6 Nov 2015 | Published 5 Jan 2016

DOI: $10.1038 /$ ncomms10153

OPEN

\title{
BPTF is required for c-MYC transcriptional activity and in vivo tumorigenesis
}

Laia Richart ${ }^{1}$, Enrique Carrillo-de Santa Pau ${ }^{1}$, Ana Río-Machín², Mónica P. de Andrés ${ }^{1}$ Juan C. Cigudosa ${ }^{2}$, Víctor J. Sánchez-Arévalo Lobo ${ }^{1} \&$ Francisco X. Real ${ }^{1,3}$

c-MYC oncogene is deregulated in most human tumours. Histone marks associated with transcriptionally active genes define high-affinity c-MYC targets. The mechanisms involved in their recognition by c-MYC are unknown. Here we report that c-MYC interacts with BPTF, a core subunit of the NURF chromatin-remodelling complex. BPTF is required for the activation of the full c-MYC transcriptional programme in fibroblasts. BPTF knockdown leads to decreased c-MYC recruitment to DNA and changes in chromatin accessibility. In Bptf-null MEFs, BPTF is necessary for c-MYC-driven proliferation, G1-S progression and replication stress, but not for c-MYC-driven apoptosis. Bioinformatics analyses unveil that BPTF levels correlate positively with c-MYC-driven transcriptional signatures. In vivo, Bptf inactivation in pre-neoplastic pancreatic acinar cells significantly delays tumour development and extends survival. Our findings uncover BPTF as a crucial c-MYC co-factor required for its biological activity and suggest that the BPTF-c-MYC axis is a potential therapeutic target in cancer.

\footnotetext{
${ }^{1}$ Epithelial Carcinogenesis Group, Cancer Cell Biology Programme, Spanish National Cancer Research Center-CNIO, Madrid 28029, Spain. ${ }^{2}$ Molecular Cytogenetics Group, Human Cancer Genetics Programme, Spanish National Cancer Research Center-CNIO, Madrid 28029, Spain. ${ }^{3}$ Departament de Ciències Experimentals i de la Salut, Universitat Pompeu Fabra, Barcelona 08003, Spain. Correspondence and requests for materials should be addressed to F.X.R. (email: preal@cnio.es) or to V.J.S.-A.L. (email: vjsanchez@cnio.es).
} 
T he MYC oncogenes encode a family of related sequencespecific bHLHZip transcription factors (c-, L- and N-MYC) that control a plethora of cellular functions including cell growth, proliferation, differentiation and apoptosis. c-MYC is deregulated in more than half of human cancers ${ }^{1}$, often in association with aggressive, poorly differentiated tumours ${ }^{2}$.

The oncogenic potential of c-MYC stems from its function as a transcriptional regulator that binds DNA on heterodimerization with MAX ${ }^{3}$. MYC-MAX heterodimers show a predilection for the palindromic 'E-box' motif (CACGTG) found in regulatory elements of genes controlled by this complex ${ }^{4}$. Nonetheless, mounting evidence indicates that the genomic distribution of c-MYC-MAX complexes is influenced by factors other than sequence specificity, most notably the chromatin context $\mathrm{t}^{5,6}$. High-affinity sites are bound by c-MYC in a wide variety of cell types and are typically enriched in $\mathrm{CpG}$ islands together with high levels of activating histone marks (H3K4me3, H3K79me, H3ac and H2A.Z). Low-affinity sites vary among cell types and are only engaged when c-MYC is expressed at high levels. Compared with high-affinity targets, they show a selective enrichment for macroH2A, H3K27me3 and H4K16ac ${ }^{5-7}$. Upon binding to its target promoters, c-MYC recruits multiple cofactors that affect the state of chromatin and the activity of RNA polymerases. Among them are chromatin-remodelling complexes (for example, SWI/SNF), acetyltransferases and methyltransferases that modify core histones (for example, P300/CBP-Associated Factor (PCAF)) and proteins associated with the basal transcriptional machinery (for example, $\mathrm{P}-\mathrm{TEFb})^{8-10}$.

The mechanisms involved in the recognition of the active chromatin configuration by c-MYC are poorly understood but likely involve the combined action of epigenetic 'readers' and chromatin remodellers that modulate the accessibility of DNA in modified nucleosomes. A plausible candidate to act as a c-MYC tethering factor is NURF (ATP-dependent nucleosome-remodelling factor), an ISWI complex that uses ATP hydrolysis to catalyse nucleosome sliding ${ }^{11,12}$. Mammalian NURF consists of three subunits: BPTF, SNF2L and pRBAP46/48. BPTF (bromodomain PHD transcription factor) provides sequence specificity to NURF through interactions with transcription factors, histone variants and histone modifications of transcriptionally active genes (H3K4me3, H4K16Ac and H2A.Z) $)^{12-14}$. We found that BPTF is mutated in bladder tumours and its knockdown in cultured bladder cancer cells results in reduced proliferation ${ }^{15}$ and hypothesized that these effects might be mediated, in part, by c-MYC.

Here we show that BPTF and c-MYC are present in a protein complex. This interaction is critical for c-MYC function, since BPTF knockdown leads to a decrease in c-MYC binding to DNA, changes in chromatin accessibility and impaired activation of the
c-MYC transcriptional programme. Consistent with this, BPTF expression in human tumours positively correlates with activation of c-MYC gene signatures. In addition, BPTF is necessary for the survival of c-MYC-overexpressing cells and for c-MYC-driven tumorigenesis in the mouse pancreas. These results highlight the potential of exploiting the BPTF-MYC axis in cancer therapy.

\section{Results}

BPTF depletion impairs c-MYC transcriptional activity. To assess whether BPTF is required for the transcriptional activity of c-MYC, human foreskin fibroblasts (HFFs) were stably transduced with the chimeric MYC-ER complementary DNA (cDNA; hereafter HFF MYC-ER) and infected with lentiviruses coding for either control (shNt) or BPTF-targeting short-hairpin RNAs (shRNAs; sh\#1 and sh\#2). To rule out proliferation-associated effects, and to avoid the interference of endogenous c-MYC, cells were driven to quiescence by serum starvation before treatment with 4-hydroxytamoxifen (4-OHT). Western blot and immunofluorescence analyses confirmed that the lentiviral shRNAs inhibited the expression of BPTF and did not interfere with either MYC-ER expression or nuclear translocation (Fig. 1a; Supplementary Fig. 1a). We examined the expression of a set of well-established c-MYC targets in control and BPTF-silenced HFF MYC-ER cells by real-time quantitative reverse transcriptase PCR (RT-qPCR). BPTF knockdown resulted in a significant impairment of the induction of 6/7 c-MYC messenger RNA (mRNA) targets with at least one of the two shRNAs (Fig. 1b; Supplementary Fig. 1b). To extend these findings, we used Bptf-null mouse embryonic fibroblasts (MEFs) ${ }^{16}$ transduced with MYC-ER. Successful recombination of the floxed allele was demonstrated by PCR and RT-qPCR analysis (Supplementary Fig. 1c,d). In these cells, addition of 4-OHT also resulted in an impaired activation of four c-MYC targets tested ${ }^{17}$ (Supplementary Fig. 1e).

To further investigate the hypothesis that BPTF is required for c-MYC-dependent transcription, we performed global RNA profiling (RNA-Seq) using HFF MYC-ER cells. Normalization with spike-in standards $^{18}$ yielded $\sim 6,700$ differentially expressed genes in 4-OHT-treated control cells relative to vehicle (Supplementary Data 1). Gene Set Enrichment Analysis (GSEA) revealed that the genes upregulated upon 4-OHT addition were significantly enriched in c-MYC-dependent transcriptional signatures ${ }^{19-21}$ and gene ontology pathways classically associated with c-MYC function (that is, ribosome biogenesis and translation, mitochondrial function, and RNA/ ribosomal RNA/transfer RNA processing). Moreover, genes downregulated in control 4-OHT-treated cells overlapped with

\footnotetext{
Figure 1 | BPTF is required for c-MYC transcriptional activity. (a) Immunofluorescence staining of BPTF and c-MYC showing MYC-ER nuclear translocation upon 4-OHT treatment in control and BPTF-silenced HFF. Scale bar, $25 \mu \mathrm{m}$ (b) Examples of expression of known c-MYC target genes, analysed by RT-qPCR, upon BPTF knockdown. Transcript levels were normalized against GAPDH and the vehicle-treated condition. Data are expressed as mean \pm s.e.m. ( $n \geq 3$ ). $P$ values were determined using an unpaired $t$-test. (c) Fold change in FPKM values of c-MYC-dependent gene sets enriched in 4OHT-treated control cells. Values are displayed for both control and BPTF-silenced HFF MYC-ER cells. Gene sets tested and $P$ values (Wilcoxon test): 1 , Schuhmacher et al. 'MYC targets up' $\left(P=3.156 \times 10^{-15}\right)$; 2 , Acosta et al. 'proliferation independent MYC targets up' $\left(P=2.034 \times 10^{-08}\right) ; 3$, Schlosser et al. 'MYC targets serum response up' $\left(P=6.62 \times 10^{-09}\right)$; 4, Schlosser et al. 'MYC targets serum response Dn' $\left(P=6.11 \cdot 10^{-09}\right)$; 5, Schlosser et al. 'MYC targets repressed by serum' $\left(P=2.747 \cdot 10^{-14}\right)$. (d) Fold change in mRNA levels for the set of 20 genes used for validation. Left panel: data calculated from FPKM values. Right panel: data calculated from $\geq 3$ independent experiments assessed by RT-qPCR. $P$ values were determined using a Wilcoxon test. (e) Examples of genes included in the validation. Transcript levels were normalized against GAPDH and the vehicle-treated condition. Data are expressed as the mean \pm s.e.m. $P$ value was determined using an unpaired $t$-test. (f) Co-immunoprecipitation of Flag-BPTF with HA-tagged c-MYC from lysates of transiently transfected 293T cells; western blotting with the indicated antibodies. (g) Endogenous BPTF and c-MYC interact in MIA PaCa-2 cells as shown by in situ proximity ligation assay. The interaction events are visible as red dots (nuclear staining in blue) and are marked by arrowheads. The interaction of MYC with MAX is shown as a positive control. Number of dots per nuclei was quantified manually $\left(n=70\right.$ nuclei). Scale bar, $5 \mu$ m. ${ }^{\star} P<0.05$; ${ }^{\star \star} P<0.01$; ${ }^{* \star *} P<0.001$.
} 
gene sets known to be repressed by c-MYC ${ }^{22,23}$ (Supplementary Fig. 1f; Supplementary Data 2). These findings are consistent with the specific transcriptional regulation of the c-MYC signature and validate the approach used.

BPTF knockdown in HFF MYC-ER cells resulted in a reduced transcriptional response to 4-OHT, both up- and downregulated genes being significantly affected (Supplementary Fig. 1g). The mechanisms involved in c-MYC-mediated repression have not been fully elucidated; therefore, we focused on its best-established role as a transcriptional activator ${ }^{18}$. We found an impaired activation of five independent c-MYC signatures in BPTF-silenced cells (Fig. 1c). These results were validated by RT-qPCR for an additional 20 genes, 19 of which are c-MYC chromatin immunoprecipitation sequencing (ChIP-Seq) targets a

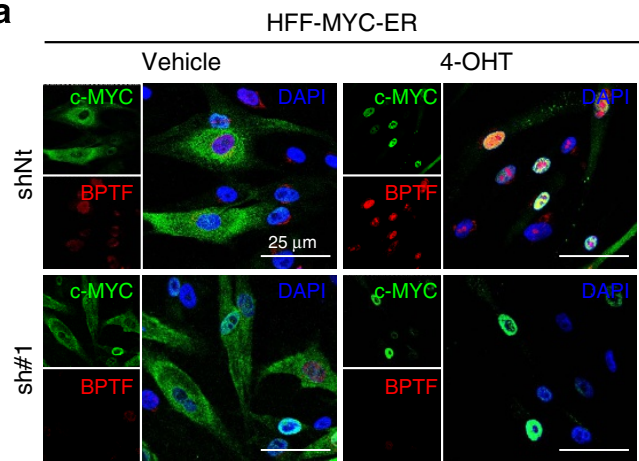

c

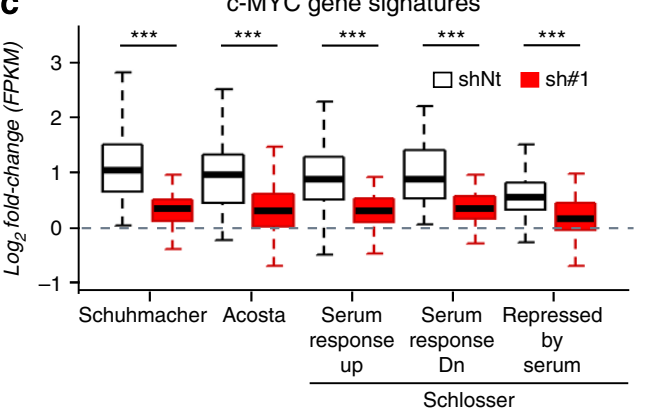

b

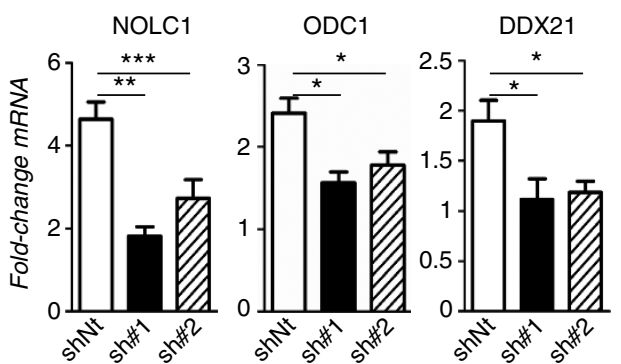

d
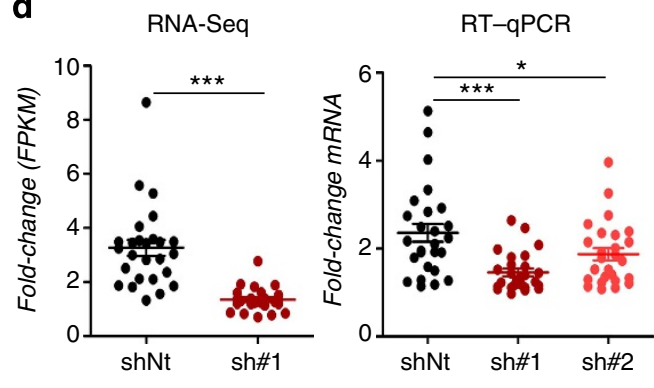

e
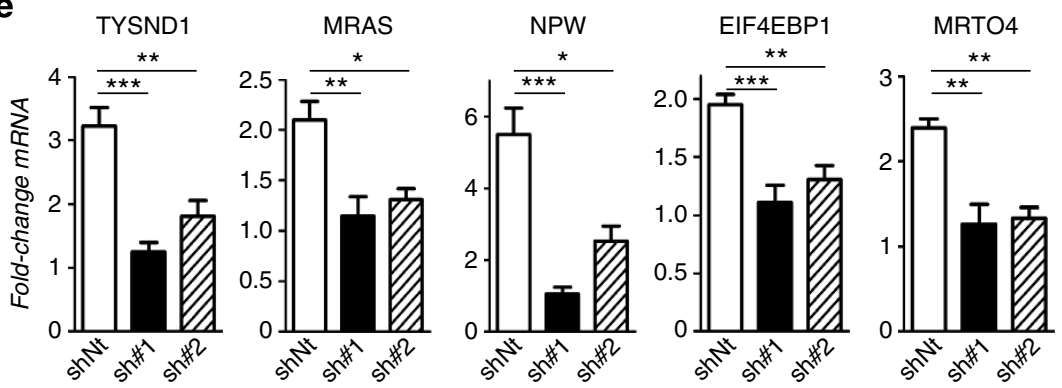

RNF207

$\mathbf{f}$

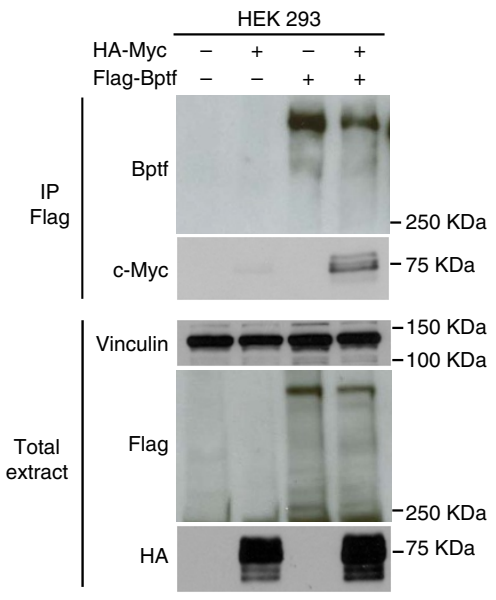

g

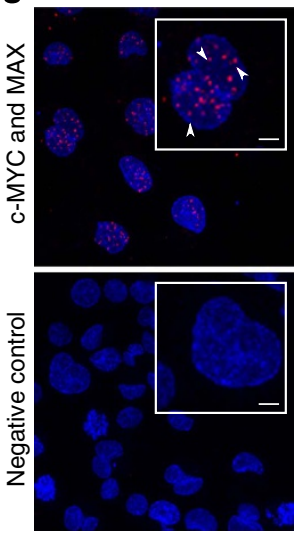

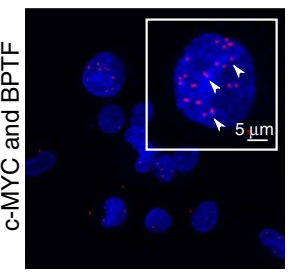

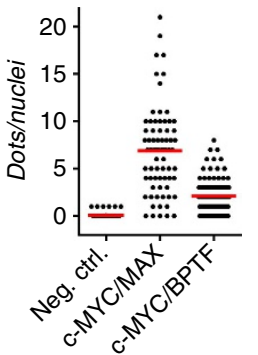


in at least one cell line profiled by ENCODE (Supplementary Data 1 and 3). The extent of induction of these genes was significantly reduced in HFF MYC-ER cells transduced with both BPTF-targeting shRNA lentiviruses (average fold change (4-OHT versus vehicle) for shNt, 2.44; sh\#1, 1.52; sh\#2, 1.97; shNt versus sh\#1, $P<0.001$; shNt versus sh\#2, $P=0.049$; unpaired $t$-test; Fig. 1d). Representative results are shown in Fig. 1e. These data indicate that BPTF is required for c-MYC-mediated gene regulation.

BPTF interacts with c-MYC. To activate transcription, c-MYC recruits chromatin-modifying and-remodelling complexes that hyperacetylate histones, displace nucleosomes and increase DNA accessibility at target regions ${ }^{8,24,25}$. BPTF recognizes histone marks present in both high- and low-affinity c-MYC target promoters and is involved in chromatin remodelling. We thus reasoned that BPTF association with c-MYC might mechanistically explain the suppression on c-MYC transcription. Immunoprecipitation of Flag-BPTF followed by western blotting to detect HA-MYC from transfected 293T lysates revealed both proteins in the same complex (Fig. 1f). The interaction between endogenous c-MYC and BPTF was validated in MIA $\mathrm{PaCa}-2$ pancreas cancer cells, expressing high levels of both proteins, using the in situ proximity ligation assay (isPLA) and affinitypurified rabbit antibodies recognizing BPTF residues 913-942 (Fig. 1g; Supplementary Fig. 2a,b). Together, these data suggest that BPTF interacts with c-MYC.

BPTF regulates c-MYC binding to DNA. The E-box motif recognized by c-MYC is very widely represented at the genomewide level and the findings described above suggested that BPTF serves to recruit c-MYC to its target promoters through its ability to recognize specific histone marks. To test this hypothesis, we conducted ChIP with anti-c-MYC antibodies followed by massive parallel sequencing (ChIP-Seq) in HFF MYC-ER cells (Supplementary Data 4). A total of 1,397 peaks were identified in 4-OHT-treated control cells. In agreement with previous reports, the analysis of the density profiles of the distance between the summit of peaks and transcription start sites (TSSs) showed that c-MYC-binding sites concentrate around the TSS (Supplementary Fig. $3 a)^{5,26}$. ChIP peaks occurred within promoter regions (TSS $\pm 3 \mathrm{~kb} ; 35.6 \%$ ), gene bodies (intragenic; $25 \%$ ) and further upstream or downstream (intergenic; 39.4\%). Sequence analysis of c-MYC-bound regions with MEME $^{27}$ unveiled a significant over-representation of the MYC-MAX-binding motif $\left(P=2.8 \times 10^{-69}\right.$; Supplementary Fig. $\left.3 \mathrm{~b}\right)$. Moreover, GSEA analysis of c-MYC-bound promoters showed highly statistically significant overlap with three transcriptional signatures of c-MYC-dependent genes and with biological modules associated with c-MYC function (for example, cell proliferation; Supplementary Fig. 3c; Supplementary Data 5). Furthermore, genes directly bound by c-MYC showed a significantly higher mRNA induction than those lacking a ChIP-Seq peak (Supplementary Fig. 3d).

To determine whether BPTF silencing interfered with c-MYC recruitment to chromatin, we analysed the magnitude and distribution of c-MYC ChIP-Seq peaks upon BPTF knockdown. Globally, c-MYC-binding intensity was significantly lower in shBPTF-expressing cells $\left(P<2.2 \times 10^{-16}\right.$, Wilcoxon test (Fig. 2a,b). This reduction was not uniformly distributed at the genome-wide level: only $50.2 \%$ of the peaks showed a read number fold change $\geq 2$ and this was unrelated to peak intensity, suggesting that the differences did not result from an inefficient ChIP. These results indicate that BPTF silencing selectively affects a subset of c-MYC ChIP-Seq peaks. We validated these observations by ChIP-qPCR on gene promoters for which a peak was identified in the ChIP-Seq experiment ('target'), as well as on 'non-target' control genomic regions (Supplementary Data 3 and 6), in at least three independent experiments. By this approach, c-MYC recruitment to target genes was significantly reduced in cells infected with the BPTF-targeting shRNAs (Fig. 2c,d; Supplementary Fig. 3e).

c-MYC target regions cluster into 'high-affinity' or 'lowaffinity' sites. We investigated the effect of BPTF knockdown on c-MYC recruitment to both types of targets, as previously defined $^{5,26}$, and observed a significant enrichment of high-affinity sites among the genes less affected by BPTF silencing (Fig. 2e,f). These data suggest that BPTF requirement for target recognition by c-MYC depends on the epigenetic context: while dispensable for c-MYC binding to $\mathrm{H} 3 \mathrm{~K} 4 \mathrm{me} 3$-rich 'high-affinity' promoters, it is necessary for c-MYC binding to low-affinity sequences, possibly through the recognition of H4K16ac. The effect of BPTF silencing on the induction of c-MYC target mRNAs was independent from the extent of reduction in c-MYC binding at their promoters (Fig. 2f; Supplementary Data 7), suggesting that c-MYC requires BPTF to activate transcription at low-affinity targets genes.

BPTF is required for c-MYC-induced chromatin remodelling To assess whether BPTF knockdown led to changes in DNA accessibility, we performed quantitative DNAse I hypersensitivity assays, as described earlier ${ }^{28}$. DNAse I hypersensitive sites mark cis-regulatory elements (that is, enhancers or promoters) and result from the cooperative binding of transcription factors and chromatin-remodelling complexes ${ }^{29}$. We analysed the DNA accessibility of c-MYC 'target' promoters validated in Fig. 2c, in the presence or absence of 4-OHT, and included 'non-target' regions for comparison. 4-OHT addition to HFF MYC-ER cells led to an increased sensitivity to DNAse I of 'target' regions in control $(P<0.001$, paired $t$-test) but not in BPTF-silenced cells (Fig. 3a (top); Supplementary Data 8). There was no consistent effect on 'non-target' promoters (Fig. 3a (bottom) and Supplementary Data 8). Overall, these results indicate that attenuation of the $\mathrm{c}-\mathrm{MYC}$ transcriptional response resulting from BPTF knockdown is associated with changes in DNA accessibility, suggesting that BPTF is necessary for the c-MYC-induced remodelling of target chromatin. We next analysed the levels of acetylated $\mathrm{H} 3$ and $\mathrm{H} 3 \mathrm{~K} 4 \mathrm{me} 3$ in 'target' and 'non-target' promoters by ChIP-qPCR. As reported previously, c-MYC activation in control cells was associated with the selective hyperacetylation of histone $\mathrm{H} 3$ in 'target' promoters $(P=0.002$, paired $t$-test). Importantly, this effect was lost upon BPTF knockdown (Fig. 3b, top). By contrast, the levels of $\mathrm{H} 3 \mathrm{~K} 4 \mathrm{me} 3$ were unaffected by BPTF silencing (Fig. 3b, middle). c-MYC activation in control cells was associated with a significant decrease in total $\mathrm{H} 3$ at $\mathrm{c}-\mathrm{MYC}$ target promoters, suggesting chromatin remodelling. BPTF silencing did not alter the basal levels of $\mathrm{H} 3$ at these sites but it impaired $\mathrm{H} 3$ depletion (Fig. 3b, bottom), in agreement with the DNAse I hypersensitivity data. In summary, these results indicate that BPTF regulates chromatin accessibility at c-MYC target promoters.

BPTF is required for a subset of c-MYC biological functions. MYC proteins regulate a wide variety of biological processes including cell proliferation, differentiation and apoptosis. Physiological c-MYC levels induce DNA synthesis through the transcriptional activation of cell cycle-related genes ${ }^{30}$ and by modulating the activity of DNA replication origins ${ }^{31}$. Here we analyse the requirement of BPTF for c-MYC-induced proliferation, replication stress and apoptosis. 
a

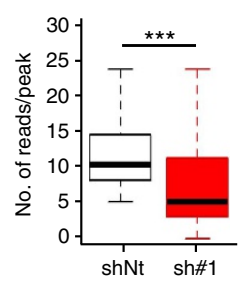

b

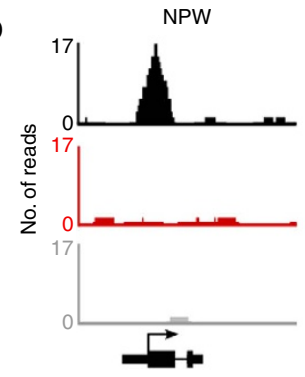

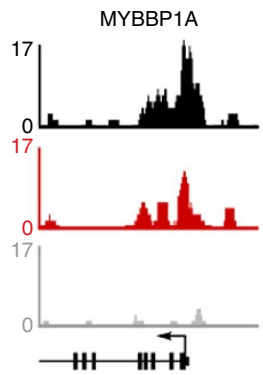

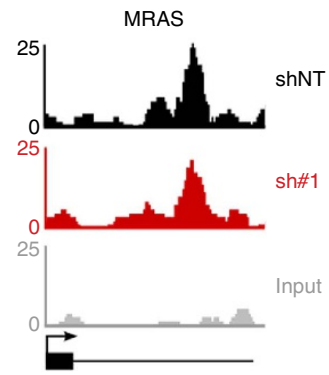

C

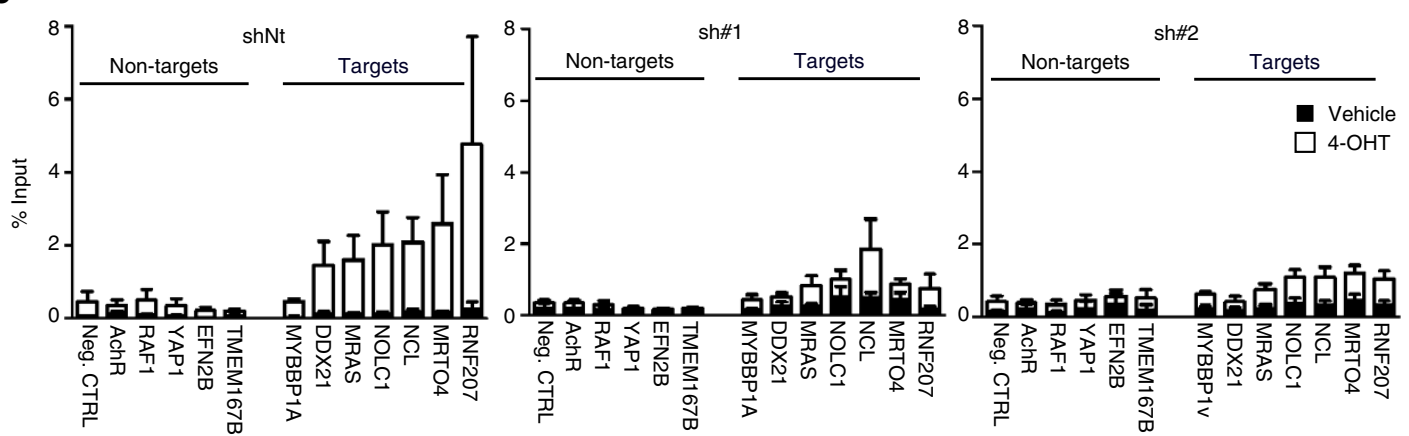

d

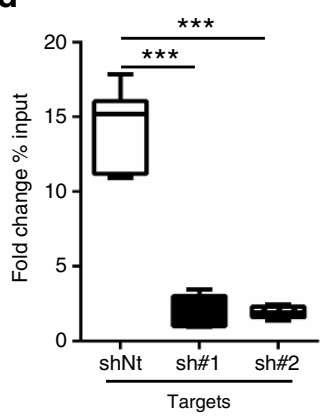

e

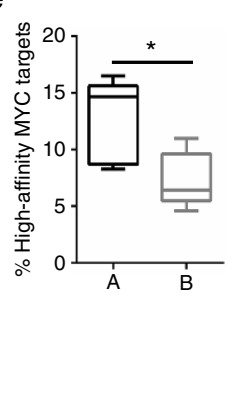

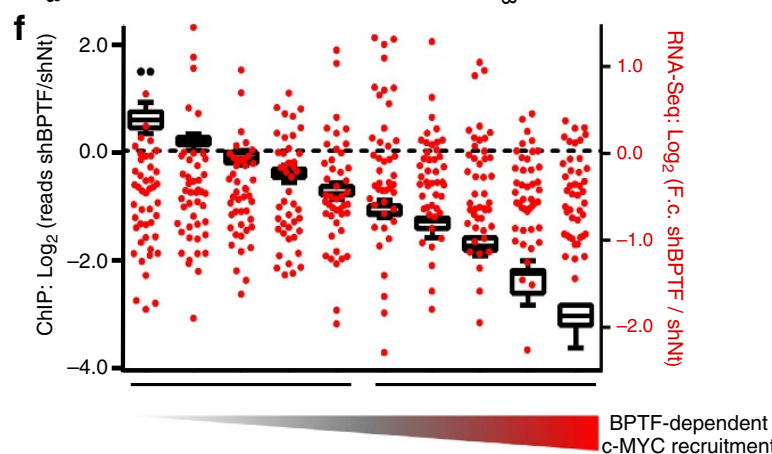

Figure 2 | BPTF silencing interferes with c-MYC recruitment to its target genes. (a) Box plot showing the intensity of c-MYC ChIP-Seq signal (reads per peak) at MYC-enriched regions in control and BPTF-silenced HFF MYC-ER cells. MYC-enriched regions were defined in 4-OHT-treated control cells. c-MYC-binding intensity was measured as number of reads per peak. ${ }^{\star \star \star} P<0.001$ (Wilcoxon test). (b) Representative snapshots of $c-M Y C$-bound genomic regions in control and BPTF-silenced HFF MYC-ER cells after stimulation with 4-OHT. (c) ChIP analysis of c-MYC enrichment at the promoters of 'target' and 'non-target' genes in control and BPTF-silenced HFF MYC-ER cells in the presence (white) or absence of 4-OHT (black). ChIP values are expressed as average \pm s.e.m. of $\%$ input chromatin. An isotype-matched lgG antibody was used as control (Supplementary Fig. $3 f$ ) and $\geq 3$ independent experiments were analysed per promoter region. (d) Fold change in \% of input following 4-OHT addition, averaged for the two different promoter populations in control and BPTF-silenced cells. ${ }^{\star \star \star} P<0.001$ (unpaired $t$-test). (e) Groups A and B are defined as in $\mathbf{f}$. High-affinity MYC targets are significantly enriched among the genes for which MYC recruitment is less affected by BPTF knockdown. ${ }^{\star} P<0.05$ (unpaired $t$-test). (f) $c-M Y C$ target genes ranked according to the change in c-MYC binding at their promoters after BPTF silencing. BPTF dependency of c-MYC recruitment to DNA is calculated as the Log 2 (reads shBPTF/reads shNt; left $y$ axis). For the same collection of ranked genes, the transcriptional response to 4-OHT is shown (scatter plot right $y$ axis). BPTF dependency of 4-OHT-dependent mRNA induction is calculated as the $\log _{2}$ (F.c. shBPTF/F.c. shNt). Four data points are outside the right $y$ axis limits.

To determine whether BPTF is required for c-MYC-induced proliferation, we used wild-type (WT) and Bptf-null MEFs. Cells were co-infected with lentiviruses coding for Cre recombinase and the MYC-ER fusion protein. Quiescent MEFs were induced to re-enter the cell cycle by adding fetal bovine serum (FBS) \pm 4 OHT and S-phase entry was assessed by 5-bromodeoxyuridine (BrdU) uptake. 4-OHT-treated WT and Bptf-null cells showed a significantly higher percentage of cells in S-phase than vehicletreated cells as early as $9 \mathrm{~h}$ after FBS $+4-\mathrm{OHT}$ stimulation $(P=0.014$, paired $t$-test $)$, indicative of MYC-induced G1/S progression (Fig. 4a). DNA content analysis of $\mathrm{BrdU}^{+}$cells followed by quantification of cells in early S-phase showed that BPTF deletion resulted in a significantly delayed progression through S-phase in MYC-ER-activated cells (at $18 \mathrm{~h}, P<0.001$, unpaired $t$-test). There were no effects on vehicle-treated cells (Fig. 4b,c).
c-MYC overexpression and/or deregulation is associated with unscheduled firing of DNA replication origins, DNA damage response and checkpoint activation ${ }^{32}$. In WT MYC-ER MEFs, 4-OHT addition led to an increase in the median signal intensity of pan-nuclear $\gamma \mathrm{H} 2 \mathrm{AX}(P=0.017$, paired $t$-test), indicative of replication stress. In Bptf-null cells, a slight increase was observed in basal conditions but there was no significant change upon addition of 4-OHT (Fig. 4d).

c-MYC can induce apoptosis when expressed from an ectopic promoter in the presence of limiting survival signals or upon stress $^{33}$. To assess whether BPTF is required for MYC-induced apoptosis, WT and Bptf-null MYC-ER MEFs were seeded at high density and cultured in $0.5 \%$ FBS containing either vehicle or 4-OHT. Apoptosis was quantified by Annexin V staining and 4,6-diamidino-2-phenylindole (DAPI) exclusion. MYC-ER activation triggered a robust apoptotic response both in WT 

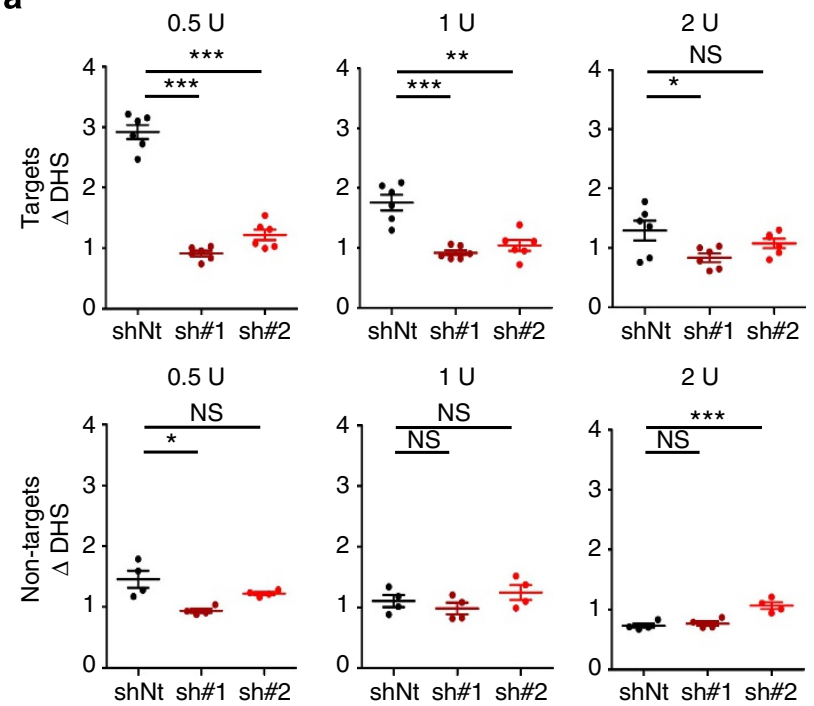

b
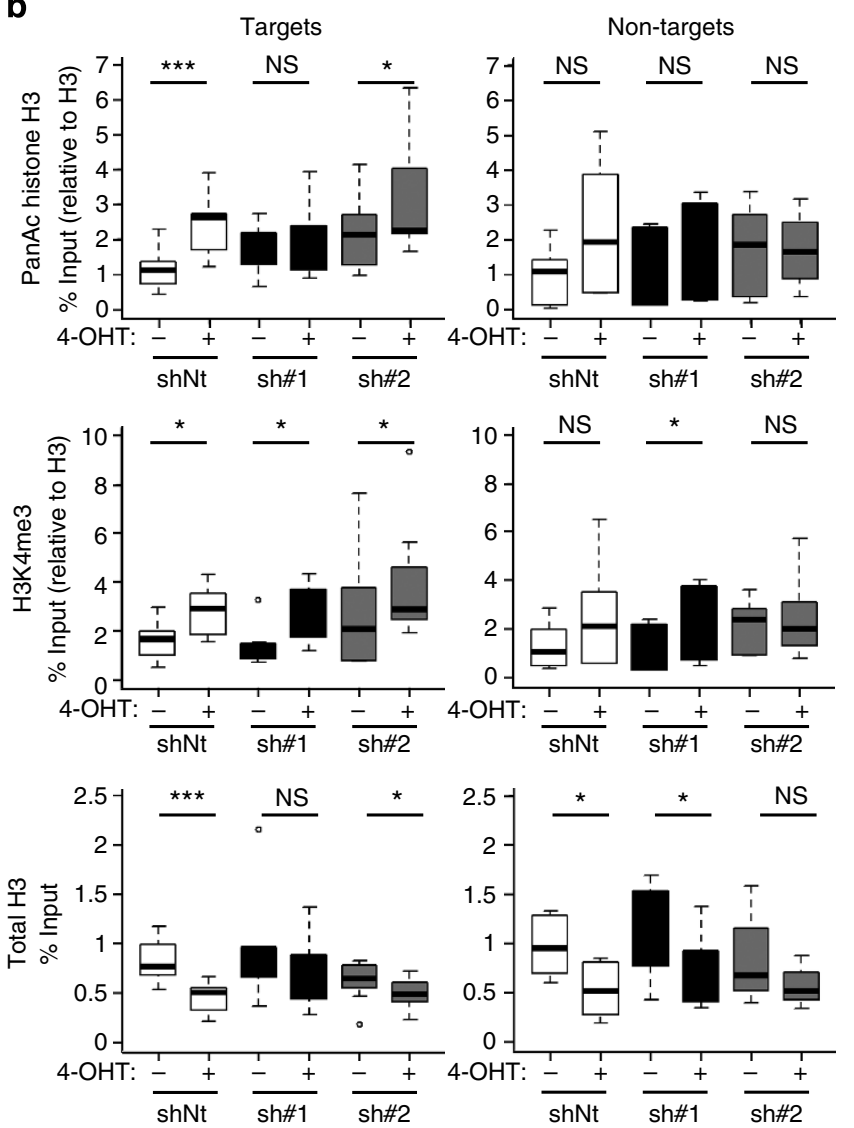

Figure 3 | BPTF is required for MYC-induced chromatin remodelling.

(a) DNase I hypersensitivity (DHS) at MYC-bound regions in control and BPTF-silenced HFF MYC-ER cells, determined by enzyme titration. Dots represent the average values of seven independent experiments. $P$ values were determined using paired $t$-test. (b) ChIP analysis of $\mathrm{Pan} \mathrm{AcH} 3$, $\mathrm{H} 3 \mathrm{~K} 4 \mathrm{me} 3$ and total $\mathrm{H} 3$ levels at the promoter of 'target' and 'non-target' genes in control and BPTF-silenced HFF MYC-ER cells. Each gene was assayed in at least three independent experiments. ChIP values are expressed as \% of input and normalized for total histone H3 (with the exception of total $\mathrm{H} 3$ ). $P$ values were determined using paired $t$-test. ${ }^{\star} P<0.05 ;{ }^{\star \star} P<0.01 ;{ }^{\star \star \star} P<0.001$. and in Bptf-null MEFs (Fig. 4e), indicating that BPTF is differentially required for a subset of c-MYC biological functions.

Deregulation of the c-MYC:BPTF axis in human cancer. To gain insight into the role of the c-MYC:BPTF axis in human cancer, we analysed public omics data sets. First, we compared BPTF and c-MYC levels with the activation of c-MYC signatures in a collection of 20 expression data sets from Oncomine and Gene Expression Omnibus (GEO) encompassing human tumours of diverse origin (Supplementary Data 9) driven by different MYC family members: Burkitt lymphoma (BL), colorectal, prostate and pancreatic tumours are mainly driven by c-MYC ${ }^{34-36}$, whereas medulloblastoma and ovarian carcinoma commonly show amplification and/or overexpression of N-MYC and L-MYC, respectively ${ }^{37,38}$. BPTF is overexpressed in tumours together with c-MYC and, in some cases, N-MYC and L-MYC (Supplementary Fig. 4a). Samples within each data set were rank-ordered by the mRNA levels of either BPTF, c-MYC, N-MYC or L-MYC and then interrogated by single-sample GSEA $\left(\right.$ ssGSEA) ${ }^{39}$ for enrichment of four c-MYC gene sets showing a modest degree of overlap (Fig. 5a). c-MYC signatures correlated with c-MYC expression levels in BL, colorectal, prostate and pancreatic carcinomas. In these tumours, BPTF expression levels also correlated positively with c-MYC signatures (Fig. 5b,c). By contrast, N-MYC and L-MYC expression levels correlated with c-MYC expression signatures only in medulloblastoma and ovarian carcinoma, respectively, and in these tumours the signatures correlated negatively with BPTF expression levels (Fig. 5b,c). These data suggest that BPTF is more selectively associated with the biological activity of c-MYC than with that of N-MYC or L-MYC.

On the basis of these findings, we focused on tumour types associated with c-MYC hyperactivation. First, we analysed the expression of BPTF and c-MYC in the Cancer Cell Line Encyclopedia data set. As shown in Fig. 6a, expression of both genes was positively correlated across all tumour types. We then selected BL and pancreatic ductal adenocarcinoma (PDAC) cells for further analysis. BL is a paradigm of a c-MYC-addicted human tumour resulting from chromosomal translocations placing c-MYC under the control of Ig regulatory sequences leading to c-MYC overexpression ${ }^{34}$. Consistently, BL-derived lines are among the highest expressors of both BPTF and c-MYC. PDAC-derived cell lines express intermediate levels of both genes (Fig. 6a) and their dependency from c-MYC is generally not the result of primary genetic alterations in this gene. BPTF knockdown in NAMALWA and RAJI BL cells was accompanied by reduced cell proliferation (Fig. 6b,c). Similarly, BPTF knockdown in PK9 and MIA Paca-2 cells led to reduced proliferation (Fig. 6d,e), indicating that tumour types with different c-MYC dependency require BPTF to sustain proliferation.

BPTF inactivation prolongs the survival of Ela1-Myc mice. On the basis of the important role of c-MYC in cancer, the bioinformatics analyses, and results of knockdown experiments shown above, we asked whether BPTF is also required for the oncogenic effects of c-MYC in vivo using genetic mouse models. Because there is increasing evidence that differentiation acts as a tumour suppressor mechanism in the pancreas ${ }^{40,41}$, we first analysed whether Bptf deletion in this tissue results in altered cell differentiation. We analysed the pancreas of 8-week-old mice in which Bptf was deleted at E9.5-E10.5 using Ptf1a-Cre as a deletor strain. Very efficient recombination and downregulation of the WT Bptf transcript was achieved (Fig. 7a). The pancreas of $B p t f^{-l o x / t o x} ; \mathrm{Ptfla}$ Cre mice was histologically normal and 
a

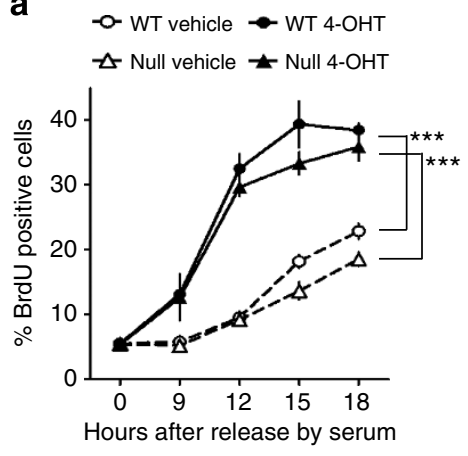

C

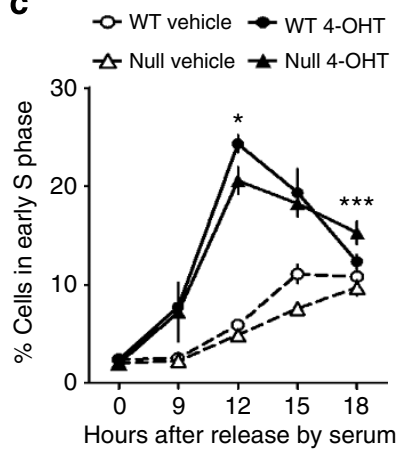

b
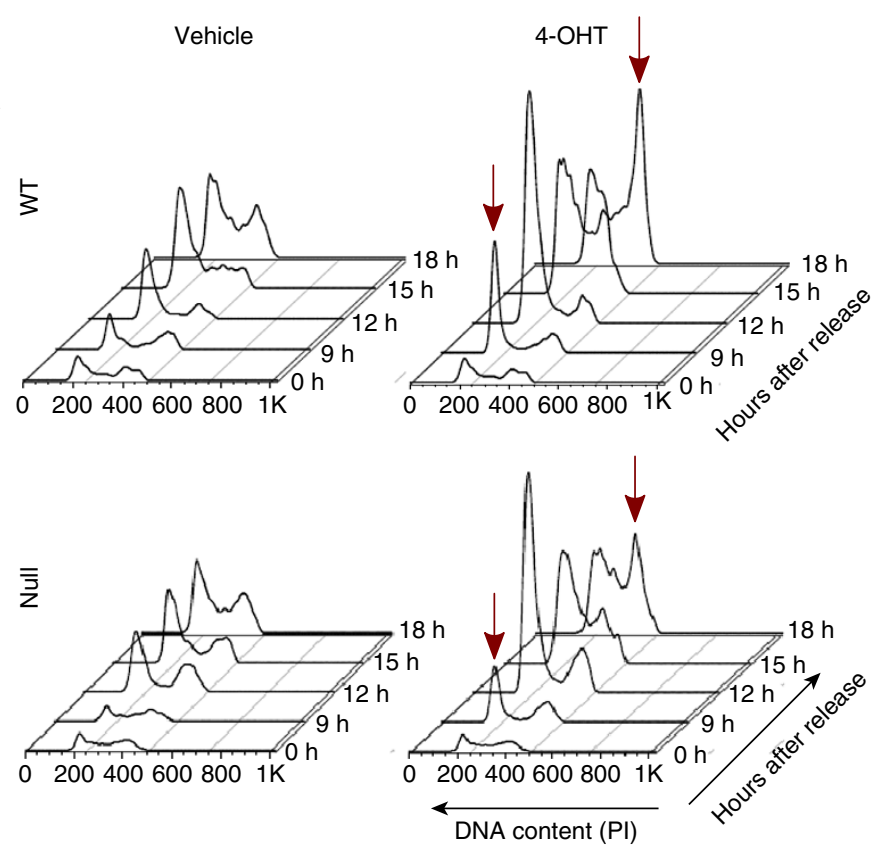

e d

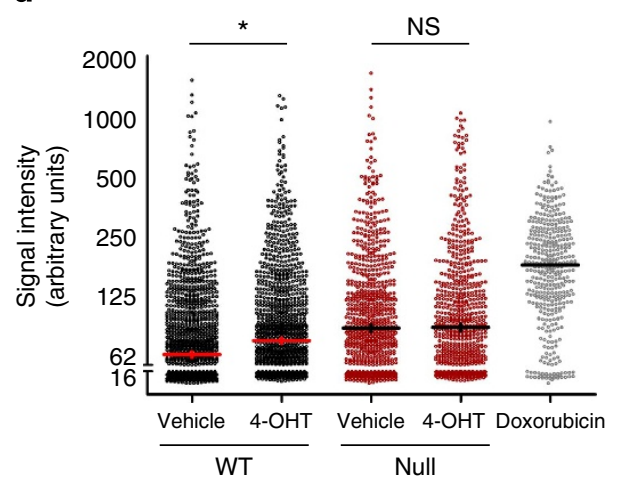

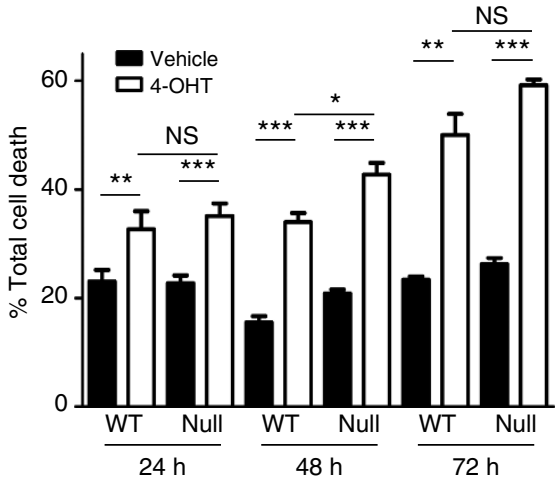

Figure 4 | Bptf is required for MYC-induced proliferation of MEFs and replication stress but not for apoptosis. (a) WT ( $n=4)$ and Bpft-null ( $n=4)$ MEFs transduced with MYC-ER were seeded at high density, arrested with $0.5 \%$ FBS for $48 \mathrm{~h}$ and stimulated with serum in the presence/absence of 4 $\mathrm{OHT}$. At the indicated time points, cells were pulse labelled with BrdU for $1 \mathrm{~h}$ before collected. Data are represented as mean $\pm s$.e.m. ${ }^{\star \star \star} P<0.001$ (paired $t$ test). (b) Histograms depicting the ploidy of BrdU-positive cells throughout the experiment described in a. (c) Quantification of early S-phase cells. The rate of loss of BrdU ${ }^{+}$early S-phase cells represents S-phase progression. Data are represented as mean \pm s.e.m. ${ }^{\star} P<0.05 ;{ }^{\star \star \star} P<0.001$ (unpaired $t$-test). (d) Replication stress-intensity of $\gamma \mathrm{H} 2 \mathrm{AX}$ signal in WT and Bpft-null MYC-ER MEFs ( $n=3$ per group) in the presence or absence of 4-OHT for $48 \mathrm{~h}$. Doxorubicin-treated cells were used as control. ${ }^{\star} P<0.05$ (paired $t$-test) (e) WT $(n \leq 4)$ and Bpft-null $(n \leq 4)$ MEFs expressing MYC-ER were seeded at high density and then transferred to $0.5 \% \mathrm{FBS}$ with or without $4-\mathrm{OHT}(2 \mu \mathrm{M})$. Apoptosis was measured as the proportion of Annexin $\mathrm{V}$-postivive cells at the indicated time points. ${ }^{\star} P<0.05$; ${ }^{\star \star} P<0.01 ;{ }^{\star \star}{ }^{\star} P<0.001$ (paired $t$-test to compare vehicle versus 4 -OHT; unpaired $t$-test to compare WT versus Bptf-null cells).

RT-qPCR analysis of acinar and endocrine markers failed to reveal evidence of altered differentiation (Fig. $7 b, c)$.

To determine the effects of Bptf deletion on tumour development/progression, we used Ela1-Myc mice where c-MYC overexpression is driven by the acinar-specific Elastase 1 promoter. These mice develop highly aggressive acinar and ductal pancreatic tumours with a $100 \%$ penetrance ${ }^{42}$. We generated Bptfox/lox;Ptf1a-CreERT2 ${ }^{+/ K I} ;$ Ela-Myc mice $\left(B p t f^{P-/-} ; E l a-M y c\right)$ where BPTF can be depleted in PTF1A ${ }^{+}$ acinar cells at will. Tamoxifen was administered orally to 5-7-week-old mice, when dysplastic acinar cells are already detectable. Oral administration of tamoxifen resulted in recombination of $60-70 \%$ of the Bptf alleles as assessed by PCR on genomic DNA (Fig. 7d). A cohort of Bptf $f^{P+/+}$;Ela1-Myc $(n=10)$, Bptf $f^{P+/-} ;$ Ela1-Myc $(n=5)$ and Bptf ${ }^{P-/-} ;$ Ela1-Myc $(n=8)$ mice was monitored weekly by ultrasound to determine tumour-free survival and animals were killed when tumour burden reached ethical end points or mice showed overt signs of morbidity. Bpt $f^{P+/+} ;$ Ela1-Myc mice displayed the expected course of pancreatic cancer onset, with a $50 \%$ disease-free survival of 13 weeks ${ }^{42,43}$. In contrast, Bptf ${ }^{P+/-} ;$ Ela1-Myc and $B p t f^{P-I-} ;$ Ela1-Myc mice showed significantly delayed tumour onset and a correspondingly delayed tumour progression (Fig. 7e,f). To further understand the molecular basis of 
a

c-MYC gene signatures

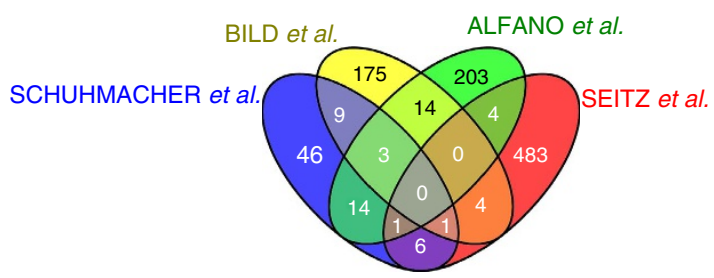

b

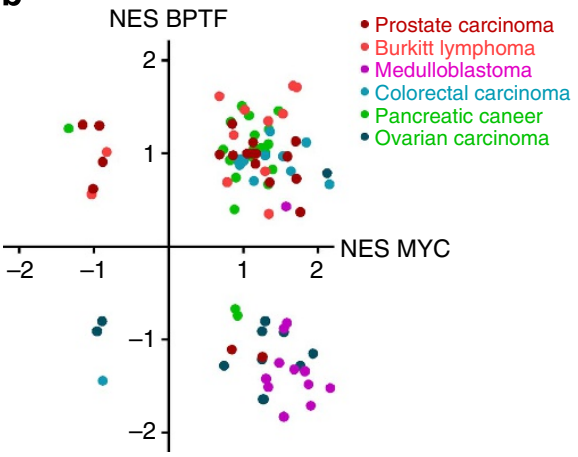

c

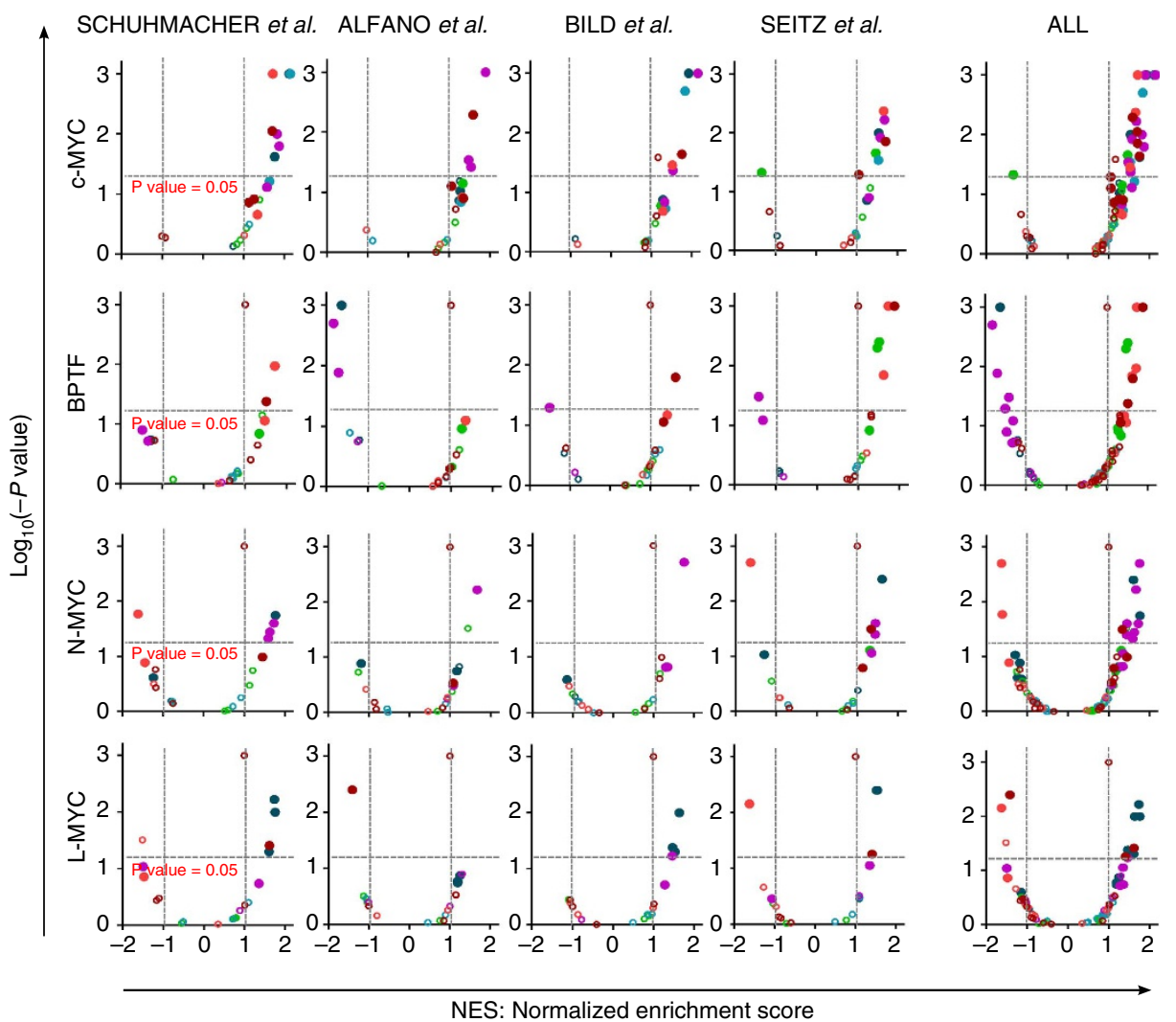

Figure 5 | BPTF expression correlates with c-MYC signatures in human tumours. (a) Venn diagram showing the overlap of the c-MYC signatures used in the following analyses. (b) Dot plot of normalized enrichment scores (NES) of the four c-MYC signatures based on GSEA. NES values were calculated for each data set previously ranked-ordered by either BPTF or c-MYC levels. (c) Volcano plots of NES and enrichment $P$ values of c-MYC signatures based on GSEA. NES values were calculated for each data set previously ranked-ordered by BPTF, c-MYC, N-MYC or L-MYC mRNA levels. Filled circles represent gene sets with a false discovery rate $<0.25$.

tumorigenesis in these mice, we analysed tumour tissues obtained at the time of death and evaluated by PCR the extent of recombination at the Bptf locus: we found evidence of tumour progression in both Bptf-deleted cells and in escaper cells in which Bptf had not been deleted (Fig. $7 \mathrm{~g}$ ). In summary, these data indicate that BPTF is necessary for the initiation and maintenance of c-MYC-driven pancreatic tumours.

\section{Discussion}

The broad range of biological activities of c-MYC, its involvement in a wide variety of human tumours, and its unique ability to regulate a large fraction of the genome have spurred a great interest in the study of the molecular mechanisms involved in the c-MYC-mediated control of gene expression.
A prominent feature of c-MYC is that sequence-specific DNA binding is only one of the requirements for its regulatory activity. Its genomic distribution is influenced by the epigenetic context, which is determined by histone modifications, histone variants and $\mathrm{CpG}$ island distribution ${ }^{5,6}$. However, how these features relate to $\mathrm{c}-\mathrm{MYC}$ engagement to promoters is not known. Here we provide evidence that BPTF interacts with c-MYC and is a key determinant of its genomic distribution and biological activity.

c-MYC target promoters can be classified according to their binding affinity and histone marks. High-affinity sites display high levels of $\mathrm{H} 3 \mathrm{~K} 4 \mathrm{me} 3, \mathrm{H} 2 \mathrm{~A} . \mathrm{Z}$ and unmethylated CpGs. Low-affinity sites are enriched in repressive histone marks (for example, H3K9me) together with $\mathrm{H} 4 \mathrm{~K} 16 \mathrm{Ac}$ and are only occupied when c-MYC levels are high ${ }^{5,6}$. BPTF can recognize 
a
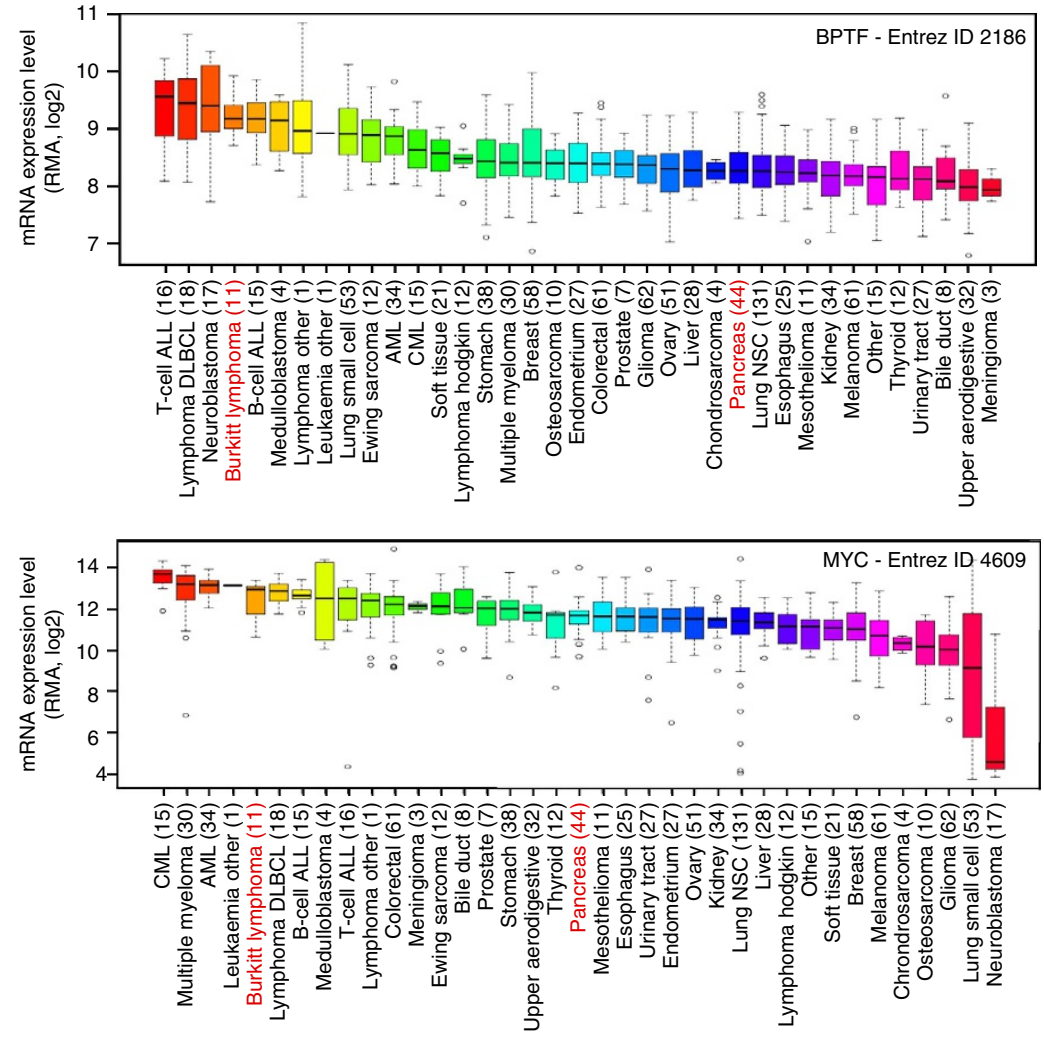

b
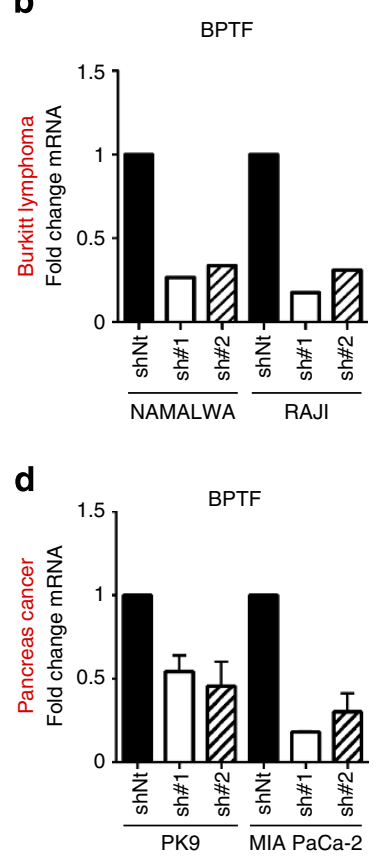

C
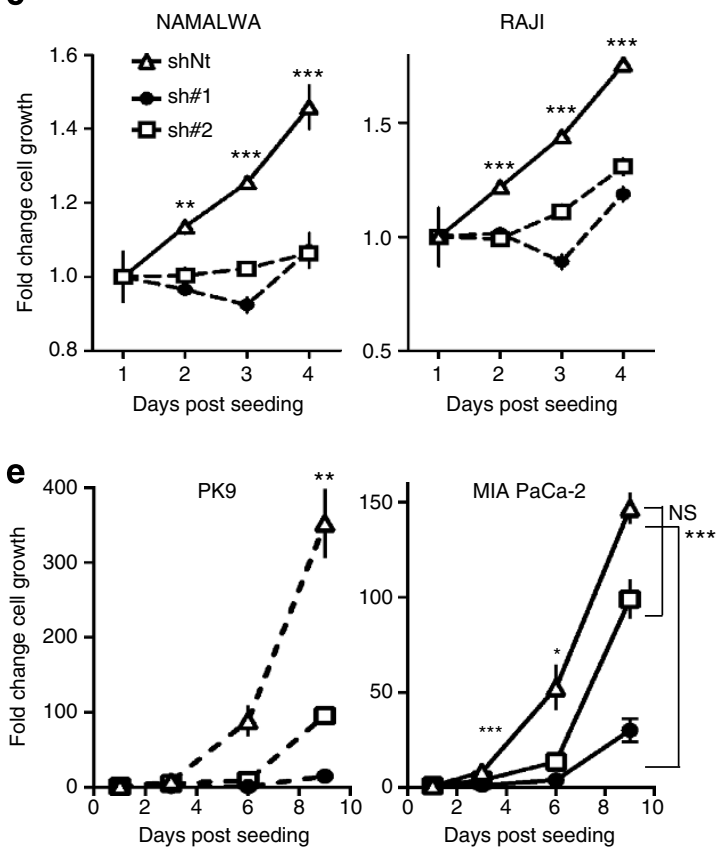

Figure 6 | BPTF knockdown suppresses the proliferation of BL and pancreatic cancer cells. (a) Top: box plot showing the relative BPTF mRNA levels across the different tumour types, extracted from CCLE_Expression_Entrez_ID_2186, with gene-centric robust multiarray analysis-normalized mRNA expression data. Bottom: box plot showing the relative c-MYC mRNA levels across a panel of human cell lines, extracted from CCLE_Expression_Entrez_ID_4609. The number of cell lines of each tumour type analysed is indicated in parentheses. Effective knockdown of BPTF in BL cells (b) and pancreatic cancer cells (d), assessed by RT-qPCR. Transcript levels were normalized against GAPDH and the sh-control samples. Proliferation analysis of two BL cell lines (NAMALWA and RAJl; c) and two pancreatic cancer cell lines (d) transduced with either control (shNt) or BPTF-targeting shRNAs (sh\#1 and \#2) $(n=5) .{ }^{\star} P<0.05 ;{ }^{\star \star} P<0.01 ;{ }^{\star \star \star} P<0.001$.

histone marks in both types of promoters; however, low-affinity promoters are more sensitive to BPTF levels. These results support the hypothesis that protein-protein interactions between
c-MYC and other chromatin-bound complexes are critical to target recognition by c-MYC ${ }^{44}$. BPTF binds $\mathrm{H} 3 \mathrm{~K} 4 \mathrm{me} 3$ through its PHD domains ${ }^{45}$ and its knockdown does not affect the levels 
a

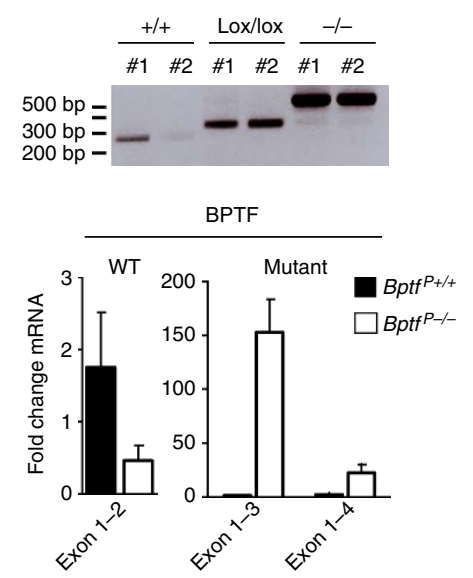

b

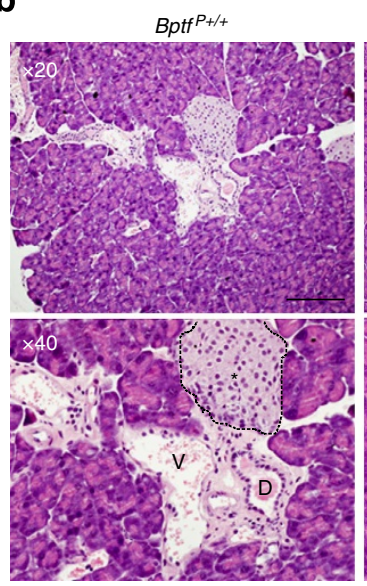

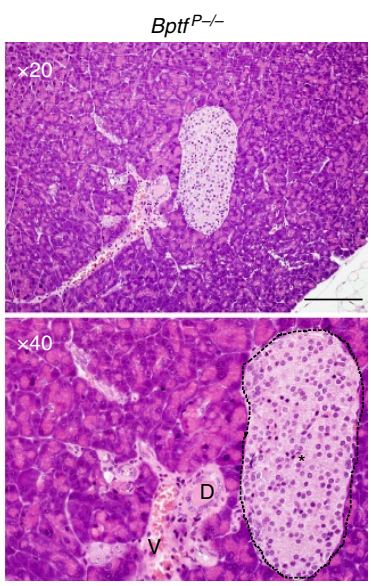

C
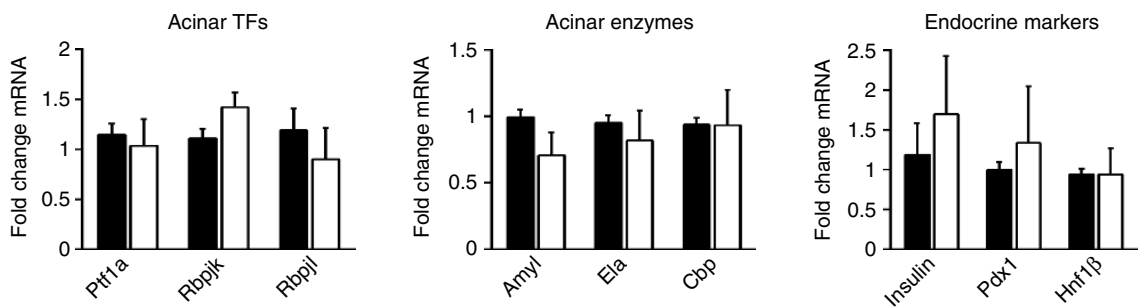

d

e
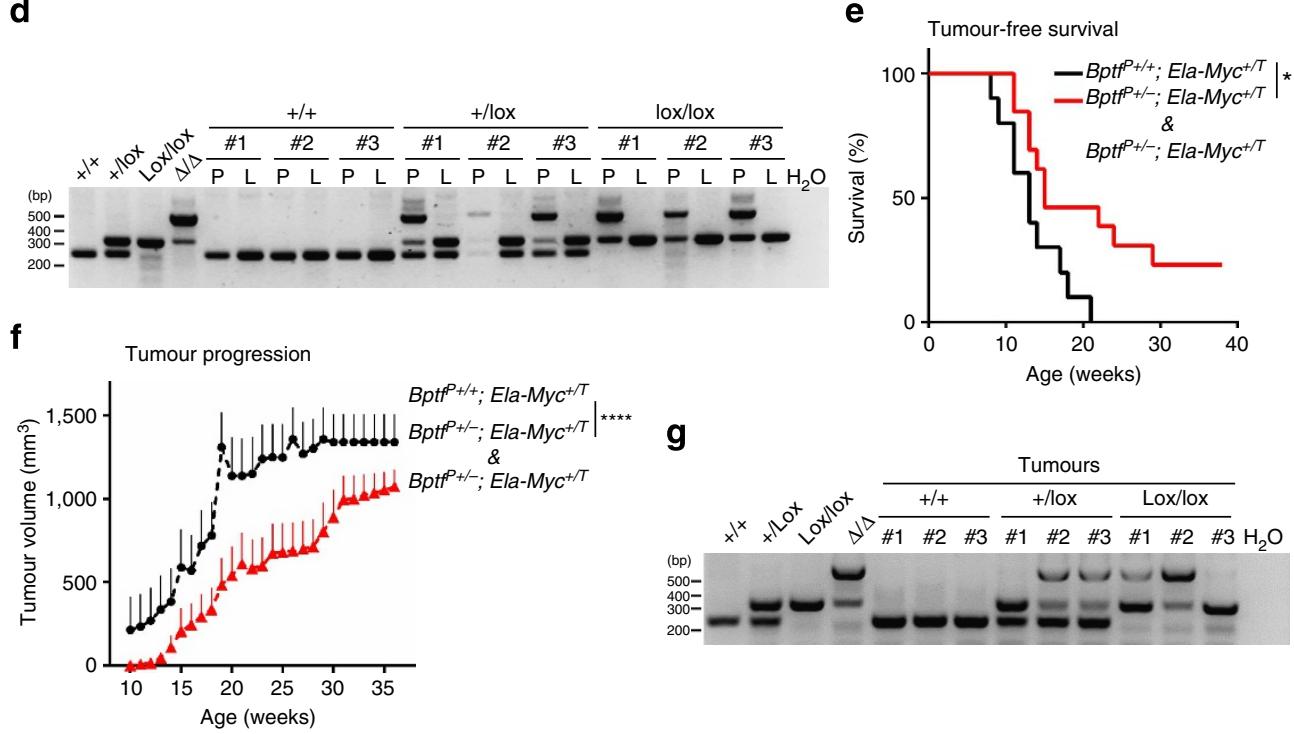

Figure 7 | BPTF is dispensable for pancreatic differentiation but its inactivation delays the onset and progression of c-MYC-driven pancreatic tumors. (a) PCR on genomic DNA showing efficient recombination at the Bptf locus in Bptf ${ }^{P-/}$ - pancreas (top). RT-qPCR analysis of BPTF WT and mutant mRNA species in control $(n=6)$ and Bptf ${ }^{P-/-}(n=7)$ mice (bottom). Transcript levels were normalized against actin and the WT condition. (b) Haematoxylineosin staining of WT and Bptf ${ }^{P-/}-$ mouse pancreatic sections. D, duct; $\mathrm{V}$, blood vessel; ${ }^{\star}$, islet of langerhans. Scale bar, $200 \mu \mathrm{m}$. (c) mRNA expression of acinar transcription factors, digestive enzymes and endocrine markers in pancreata of WT and Bpt $f^{P-/-}$ mice assessed by RT-qPCR ( $n=3$ per genotype). Transcript levels were normalized against actin and the WT condition. (d) PCR on pancreas (P) genomic DNA assessing the extent of recombination at the Bptf locus in 5-7-week-old Ptf1a-CreERT2 ${ }^{+/ K I}$;Ela-Myc mice of the corresponding genotypes. Liver (L) samples were used as negative controls.

(e) Kaplan-Meier curves of tumour-free survival are shown for Ela-Myc mice of the indicated Bptf genotypes. ${ }^{\star} P<0.05$ (log-rank test). (f) Tumour volume of Ela-Myc mice of the indicated Bptf genotypes as determined by ultrasound. Data are expressed as the mean \pm s.e.m. ${ }^{\star \star \star \star} P<0.00001$ (Wilcoxon test). (g) PCR analysis of genomic DNA from tumours arising in Bptf ${ }^{P+/+}$, Bptf $^{P-/+}$ and Bptf ${ }^{-/-}$; Ela-Myc mice.

of this histone mark at c-MYC target promoters. By contrast, BPTF silencing reduces the hyperacetylation of $\mathrm{H} 3$, commonly associated to c-MYC activation ${ }^{46}$. These data suggest that BPTF, either on its own or through its association with c-MYC, is required to recruit and/or modulate the activity of histone acetyltransferases (HATs) or histone deacetylases at promoters.
Precedents for this kind of regulation are found in Drosophila melanogaster, where Nurf301 is needed for the histone acetyl-transferase ATAC to access chromatin and maintain the decondensed architecture of the male X chromosome ${ }^{47}$. More work is required to determine how BPTF regulates the recruitment of HATs/histone deacetylases by c-MYC. 
BPTF silencing not only affected the transcriptional response of promoters where c-MYC binding was reduced but also of those where recruitment was unaffected. We propose that this results from defective chromatin remodelling, as suggested by the fact that BPTF silencing blocks the increase in DNA accessibility at c-MYC promoters typically linked to c-MYC activation. c-MYC recruits the chromatin remodeller SWI/SNF ${ }^{8}$, whose activity is partially inhibited by the linker histone $\mathrm{H} 1$ (ref. 48). NURF is necessary for $\mathrm{H} 1$ displacement at the promoters of progesterone receptor target genes ${ }^{13}$. Thus, we hypothesize that NURF-mediated eviction of $\mathrm{H} 1$ is a pre-requisite for subsequent remodelling and nucleosome eviction by SWI/SNF. Moreover, BPTF may either prevent or enhance the binding of transcription factors to DNA sequences adjacent to CCCTC-Binding Factor (CCTC)-binding sites. For example, the binding of KLF4 in the vicinity of CTCF sites is prevented by BPTF due to its nucleosome-remodelling activity 49

The recognition of active histone marks in regions of open chromatin by BPTF may facilitate and stabilize c-MYC binding. However, alternative scenarios are possible and the mechanisms through which c-MYC co-factors operate may differ at high- and low-affinity promoters. Our studies do not allow us to conclude on the sequence of recruitment of BPTF and c-MYC to target promoters. This is, in part, due to the fact that the use of RNA interference leads only to a partial reduction of BPTF levels. Therefore, it is possible that-when present at low levels-BPTF could differentially bind to histone marks and thereby affect gene expression.

Recently, WDR5 and c-MYC have been shown to interact directly. A c-MYC-WBM mutant unable to bind WDR5 showed reduced chromatin recruitment capacity, suggesting that WDR5 is important for c-MYC binding to chromatin ${ }^{10}$. A direct comparison of BPTF and WDR5 is required to determine the specific role of each of these proteins in the regulation of c-MYC activity.

The impact of BPTF silencing on c-MYC recruitment to distal enhancer elements remains to be determined. Active enhancers are characterized by high $\mathrm{H} 3 \mathrm{~K} 4 \mathrm{me} 1-2, \mathrm{H} 3 \mathrm{~K} 27 \mathrm{ac}$, recruitment of the HAT p300 and the presence of transcription factor-binding motifs and DNAse I hypersensitivity sites ${ }^{50-52}$. The C-terminal PHD finger of BPTF binds H3K4me2 (ref. 53). It is thus possible that NURF is recruited to enhancers through $\mathrm{H} 3 \mathrm{~K} 4 \mathrm{me} 2$, along with other chromatin remodellers such as CHD7 and BRG1 (refs 54,55). Furthermore, as indicated above, BPTF interacts with CTCF that can bind and regulate nucleosome distribution at enhancers $^{49}$.

c-MYC has a wide range of biological activities determined, in part, by its expression levels. Therefore, we asked for which of them is BPTF required. In multiple cell types, we show that BPTF is essential for c-MYC-driven proliferation. However, it is not necessary for MYC-triggered apoptosis in MEFs. Omomyc, a c-MYC mutant protein that cannot dimerize with MAX and does not bind E-boxes, also displays selective effects on c-MYC function: it interferes with proliferation but it exacerbates apoptosis triggered by high c-MYC levels ${ }^{56,57}$. The selective effect of BPTF knockdown on a subset of c-MYC functions could be related to the mechanisms of transcriptional activation of the genes involved. To induce cell proliferation, c-MYC binds directly the promoter of genes participating in DNA replication and cell cycle control (that is, MCM5, MCM6 or DBF4) and enhances their transcription ${ }^{26}$. By contrast, c-MYC-driven apoptosis is mostly indirect, through pathways that do not require binding to DNA, and involves the stabilization of p19 $9^{\mathrm{ARF}}$ and $\mathrm{p} 53$ or the downregulation of anti-apoptotic BCL-2 through inhibition of MIZ-1 (ref. 58). We therefore propose that
BPTF is only required for those c-MYC functions involving direct binding to chromatin.

MYC oncogenes have partially overlapping biological functions but display distinct tissue-specific expression and transforming activity ${ }^{59}$. c-MYC and N-MYC can functionally replace one another in certain contexts (for example, murine development) and share similar oncogenic potency 60,61 , but N-MYC is distinctly involved in the regulation of genes involved in neural development ${ }^{62}$. Despite that less is known on L-MYC, it appears to differ from the other family members ${ }^{22}$. Our genomic analyses suggest that BPTF may differentially affect the oncogenic activity of MYC family members and BPTF levels correlate positively with a c-MYC transcriptional signature. CTCF is required for c-MYC expression ${ }^{63}$; it is possible that BPTF and CTCF interact to activate c-MYC expression through the recruitment of transcription factors to its promoter. More work is required to determine the relevance of the epigenetic context of, and the requirement of BPTF for, the activity of N-MYC and L-MYC.

In recent years, genetic mouse models have shown that tumours become addicted to $\mathrm{c}-\mathrm{MYC}^{64}$. In inducible c-MYC-driven models, established tumours regress upon withdrawal of ectopic c-MYC expression ${ }^{65}$. Even transient inactivation of MYC is sufficient to restore checkpoint mechanisms resulting in tumour regression, remodelling of the tumour microenvironment, and shutdown of angiogenesis ${ }^{56}$. These studies also show that systemic c-MYC inhibition in mice causes a mild and reversible toxicity in proliferative tissues. On the basis of these findings, we analysed whether the in vivo oncogenic effects of c-MYC are dependent on BTPF using the Ela-Myc model of pancreatic cancer. KRAS is the main oncogene involved in human PDAC; however, c-MYC is amplified and overexpressed in $10 \%$ and $>50 \%$ of human PDAC, respectively ${ }^{66}$. The relevance of $\mathrm{c}-\mathrm{MYC}$ to pancreatic carcinogenesis has been underscored by the finding that, in mice, c-MYC is required for mutant KRas-driven development of preneoplastic pancreatic lesions and invasive tumours ${ }^{67}$. In ElaMyc mice, dysplasia is observed at 8 weeks of age and multifocal tumours develop starting a few weeks later; acinar tumours occur early while ductal tumours-reminiscent of human PDAC-appear later ${ }^{42,43}$. In this highly aggressive model, elimination of a single Bptf allele was sufficient to delay tumour initiation (that is, dysplasia) and progression. Importantly, these effects did not result from an incomplete maturation of exocrine cells-as in other systems-since we did not find evidence of altered acinar differentiation in $B p t f^{P-/}-$ mice, as determined by histological analysis and digestive enzyme mRNA expression. These findings support the notion that disruption of the BPTF-c-MYC interaction may represent a valuable strategy for the therapy of c-MYC-driven tumours. In this regard, it is noteworthy that incomplete inactivation of Bptf in acinar cells contributed to tumour development in a fraction of mice. The mechanisms through which Bptf inactivation suppresses MYC-driven tumour development in vivo need to be studied further.

Despite the in vivo genetic evidences, a therapeutic approach to target c-MYC has remained elusive. Unlike other transcription factors, c-MYC lacks a ligand-binding domain, what constitutes a formidable obstacle towards direct inhibition. Recently, the disruption of chromatin-dependent processes that suppress c-MYC activity - such as the inhibition of the BET bromodomain protein Brd4 by JQ1-has shown promising results in experimental models of multiple myeloma, BL, acute myeloid leukaemia, acute lymphoblastic leukaemia and neuroblastoma ${ }^{68,69}$. However, it is predicted that the effects of these inhibitors would be restricted to tumours where c-MYC 
transcription is dependent on BRD4. We have shown that BPTF is required for cell proliferation of $\mathrm{BL}, \mathrm{PDAC}$ and bladder cancer cells in vitro. The broad pattern of expression of BPTF in tissues ${ }^{70}$ and the correlation between BPTF expression and c-MYC signatures in human tumours suggest that a wide range of tumour types might be amenable to the therapeutic disruption of the c-MYC-BPTF axis. We therefore propose that small molecules capable of disrupting this interaction may represent a valuable approach to treat c-MYC-addicted tumours.

To conclude, we have identified BPTF as an important cofactor required for the full deployment of the biological activity of c-MYC in vitro and in vivo, including its oncogenic effects.

\section{Methods}

Generation of polyclonal anti-BPTF anti-sera. The RLHRMTSIEREEKEKVKKKEKKQEEETC peptide (residues 913-942) was chemically synthesized, coupled with keyhole limpet haemocyanin (KLH), and used as immunogen for the generation of polyclonal antibodies against BPTF. Two rabbits were inoculated subcutaneously with $500 \mu \mathrm{g}$ of peptide-KLH conjugate emulsified in Freund's complete adjuvant. Five rounds of $250 \mu \mathrm{g}$ peptide-KLH boosters were administered together with Freund's incomplete adjuvant to each animal in the interval of 3 weeks. Antibodies against BPTF were purified from serum by affinity chromatography on a HiTrap NHS-activated High Performance column (Sigma-Aldrich, GE17-0716-01, St Louis, MO, USA) and tested by enzyme-linked immunosorbent assay, in HEK293T-BPTF-Flag-transfected cells and in BPTF-silenced VM-CUB-3 cells. Uncropped western blots are provided in Supplementary Fig. 5.

Cell culture. Primary neonatal HFFs (a kind gift of M. S. Soengas, CNIO, Madrid, Spain), 293T (transformed human embryonic kidney cells, from ATCC, Rockville, MD, USA) and human adherent cancer cells-MIA PaCa-2 (from ATCC), PK9 (pancreas, from C. Iacobuzio-Donahue, Memorial Sloan-Kettering Cancer Center, New York, NY, USA) and VM-CUB-3 (bladder, L. J. Old, Memorial Sloan-Kettering Cancer Center) - were cultured in DMEM (Sigma-Aldrich) supplemented with 10\% FBS (HyClone, Logan, UT, USA), sodium pyruvate (Life Technologies, Madrid, Spain) and penicillin/streptomycin (Life Technologies). Mouse Bptf $f^{+1+}$ and Bpt $f^{\text {dox/lox }}$ MEFs were cultured in DMEM supplemented with $10 \%$ FBS, sodium pyruvate, non-essential amino acids (Life Technologies), $\beta$-mercaptoethanol (Sigma-Aldrich) and penicillin/ streptomycin. NAMALWA and RAJI cells (from ATCC) were cultured in suspension in RPMI medium (Sigma-Aldrich) supplemented with 10\% FBS and penicillin/streptomycin.

MEFs were generated by mechanical disruption and trypsin digestion of E13.5 embryos from which the fetal liver and the head had been removed. Recombination efficiency of exon 2 upon Cre recombinase expression was evaluated by PCR on genomic DNA as reported elsewhere ${ }^{1}$ (Supplementary Fig. 1c). The following primers were used: $5^{\prime}$-CTCAGGAATTAAGAGGTAATTGACTATC- ${ }^{\prime}, 5^{\prime}$-TG ATTTAGTTCTGATTGTTAGGTCTAC- ${ }^{\prime}$ and $5^{\prime}$-AGACCAGCCTGTTCTACAT GGCCAGCC- $3^{\prime}$

Animal experiments. The following mouse strains were used: $B p t f^{d o x / l o x}$ (ref. 16), Ptfla-Cre (ref. 71), Ptfla-CreERT2 ${ }^{+/ K I}$ (ref. 72) and Ela1-Myc (ref. 42). C57BL/6 $B p t f^{\text {ox } / l o x}$ mice were obtained from Jackson Laboratories (stock number 009367). Other strains were available at CNIO.

To inactivate Bptf to analyse its role in c-MYC-driven pancreatic tumorigenesis, we administered $25 \mathrm{mg}$ of tamoxifen (Sigma-Aldrich, T-5648) by gavage over the course of 1 week to 5-7-week-old Bptfox/lox; Ptf1a-CreERT2 ${ }^{+/ K I}$;Ela1-Myc mice and their corresponding controls. Mice were screened for pancreatic tumours once a week using a small animal ultrasound system.

Mice were housed under specific pathogen-free conditions according to institutional guidelines. Mice were observed on a daily basis and killed when they showed signs of morbidity or tumour burden was $>10 \%$ body weight in accordance with the Guidelines for Human Endpoints for Animals Used in Biomedical Research. All experiments were performed in accordance with the guidelines for Ethical Conduct in the Care and Use of Animals as stated in The International Guiding Principles for Biomedical Research involving Animals, developed by the Council for International Organizations of Medical Sciences (CIOMS), and were approved by the Instituto de Salud Carlos III Ethical Committee.

Plasmids and lentiviral infections. Mission shRNAs (Sigma-Aldrich) were used for RNA interference. Two BPTF-targeting shRNAs (shBPTF-1, clone TRCN0000016819; shBPTF-2, clone TRCN0000016820) were used and compared with a control non-targeting shRNA. MYC-ER was expressed from the cDNA cloned in FG12 plasmid. For lentiviral transduction of Cre recombinase, we used the lentiviral vector pLVXpuro-iCRE-ORF, a gift from C. Bar and M.A. Blasco (CNIO).

Infectious lentiviruses were produced in $293 \mathrm{~T}$ cells by calcium phosphatemediated transfection of the lentiviral construct together with the packaging plasmids psPAX2 and pCMV-VSV-G. Post transfection $(48 \mathrm{~h})$, the medium was collected twice for an additional $48 \mathrm{~h}$. Viral supernatants were filtered and either frozen down in aliquots or applied on target cells in the presence of $5 \mu \mathrm{g} \mathrm{ml}^{-1}$ polybrene. Cells were used after $48 \mathrm{~h}$ puromycin selection $\left(2 \mu \mathrm{g} \mathrm{ml}^{-1}\right)$. Human fibroblasts were infected first with lentivirus coding for MYC-ER, expanded and then infected with either control or BPTF-targeting shRNAs. MEFs were infected concomitantly with lentivirus encoding for MYC-ER and Cre recombinase.

Viability assays of BL cell lines. BL cells $\left(3 \times 10^{5}\right.$ cells per well) were seeded on plastic plates coated with retronectin (Fisher Scientific, Pittsburgh, PA, USA) and preloaded with viral supernatants. After three additional rounds of infection with viral supernatants supplemented with polybrene $\left(8 \mu \mathrm{g} \mathrm{ml}^{-1}\right)$, cells were allowed to recover for $24 \mathrm{~h}$, then selected for $48 \mathrm{~h}$ in puromycin-containing medium $\left(2 \mu \mathrm{g} \mathrm{ml}^{-1}\right)$. After selection, cells ( $\mathrm{sh} \# 1, \mathrm{sh} \# 2$ and shNT) were plated $\left(5 \times 10^{3}\right.$ per well in 96 -well plates) in replicates. Viable cell count was assessed at the indicated time points by adding WST1 cell proliferation reagent (Roche, Basel, Switzerland) to each well and determining $\mathrm{OD}_{450 \mathrm{~nm}}$ after $2 \mathrm{~h}$, according to the manufacturer's instructions.

Co-immunoprecipitation analyses. 293T cells transiently transfected with the corresponding plasmids were washed twice with ice-cold PBS and lysed for $30 \mathrm{~min}$ on ice with NP-40 lysis buffer ( $50 \mathrm{mM}$ Tris: $\mathrm{HCl} \mathrm{pH} 8.0,150 \mathrm{mM} \mathrm{NaCl}$ and $1.0 \%$ NP-40) supplemented with a protease inhibitor cocktail. Lysates were then centrifuged at $16,000 \mathrm{~g}$ for $20 \mathrm{~min}$ at $4{ }^{\circ} \mathrm{C}$. Total protein $(1 \mathrm{mg})$ was incubated with primary antibody $(2 \mu \mathrm{g})$ overnight. Protein A/G agarose beads (Laboratorios Conda, Madrid, Spain) preblocked with bovine serum albumin (BSA) were then added to the lysates. Following 4 -h incubation at $4{ }^{\circ} \mathrm{C}$, beads were washed three times with NP-40 lysis buffer and immunoprecipitated proteins were eluted with SDS sample buffer by boiling at $90^{\circ} \mathrm{C}$. SDS-polyacrylamide gel electrophoresis was then performed on 6 and $10 \%(\mathrm{w} / \mathrm{v})$ gels and proteins were then transferred onto nitrocellulose membranes.

Western blotting. Cells were lysed in RIPA buffer supplemented with protease and phosphatase inhibitors. Following sonication, clearing by centrifugation, and protein determination, equal amounts of protein per sample were subjected to electrophoresis in 8 or $10 \%$ polyacrylamide SDS gels, or in NuPAGE $3-8 \%$ Tris-acetate precast polyacrylamide gels (Life Technologies). Samples were run under reducing conditions and then transferred to nitrocellulose membranes, which were blocked with TBST, $5 \%$ skim milk. Membranes were subsequently incubated with the following primary antibodies: BPTF (1:500; ab72036, Abcam, Cambridge, UK), c-MYC (1:500; 06-340, Millipore, Billerica, MA, USA) and Vinculin (1:2,000; V9131-2ML, Sigma-Aldrich). This was followed by incubation with horseradish peroxidase-conjugated secondary antibodies (1:10,000; Dako, Glostrup, Denmark). Reactions were detected using the ECL system.

Immunofluorescence staining and isPLA. Cells grown on coverslips were fixed with $4 \%$ paraformaldehyde for $10 \mathrm{~min}$, washed and permeabilized with $0.1 \%$ Triton X-100 in PBS for 10 min. Samples were washed in PBS and blocked with 3\% BSA in PBS for $1 \mathrm{~h}$ at room temperature. Primary antibody incubation was performed in blocking solution for $2 \mathrm{~h}$ at room temperature. Mouse anti-MYC (C-33, sc-42, Santa Cruz Biotechnology, Dallas, TX, USA) was used at a 1:50 dilution and home-made affinity-purified rabbit anti-BPTF antibodies were used at $10 \mu \mathrm{g} \mathrm{ml}^{-1}$ After three washes with PBS, cells were incubated with an appropriate secondary antibody diluted in blocking solution. Nuclei were counterstained with DAPI and coverslips were mounted on ProLong (Life Technologies). Images were taken with a confocal microscope, using a $\times 40$ immersion oil lens. For the isPLA, the DuolinkII fluorescence system was used (Olink Bioscience, Uppsala, Sweden).

Quantitative real-time PCR. Total RNA was isolated from cultured cells using the GenElute Mammalian Total RNA Miniprep Kit (Sigma-Aldrich) according to manufacturer's instructions. Samples were treated with DNase I before reverse transcription (Life Technologies). cDNA was generated from $1 \mu \mathrm{g}$ of RNA using random hexamers and reverse transcriptase. Real-time PCR amplification and analysis was conducted using the 7900HT Real-Time PCR System (Applied Biosystems, Life Technologies). RNA levels were normalized to GAPDH expression using the $\Delta \Delta \mathrm{Ct}$ method. For RT-qPCR analysis, primer pairs were designed to achieve product lengths of 200-250 bp. Primer sequences are provided in Supplementary Data 3.

FACS analysis of proliferation and apoptosis. For cell cycle analysis, cells were pulse labelled with $10 \mu \mathrm{M}$ of BrdU (Sigma-Aldrich) for $1 \mathrm{~h}$, collected by trypsinization and then fixed in 100\% ethanol. Upon DNA denaturation using $2 \mathrm{~N}$ $\mathrm{HCl}$, cells were stained with mouse anti-BrdU primary antibody (sc-51514, Santa 
Cruz Biotechnology; $1 \mu \mathrm{g}$ per $10^{6}$ cells) and anti-mouse Alexa Fluor 488-conjugated secondary antibody (A21202, Life Technologies; $1 \mu \mathrm{g}$ per $10^{6}$ cells). DNA was stained by resuspending cells in $0.1 \mathrm{mg} \mathrm{ml}^{-1}$ of propidium iodide and incubating for $30 \mathrm{~min}$ at room temperature until fluorescence-activated cell sorting (FACS) analysis.

To measure apoptosis, MEFs were seeded at high density and then transferred to $0.5 \%$ FBS-containing DMEM in the presence of either vehicle (EtOH) or $2 \mathrm{mM}$ 4-OHT. At the indicated time points, cells and supernatants were collected, washed and resuspended in Annexin V-binding buffer containing $5 \mu \mathrm{l}$ per sample of Annexin V-APC (550474, BD Biosciences, Franklin Lakes, NJ, USA). DAPI was added before analysis.

All samples were analysed using a FACS Canto II (BD Biosciences) flow cytometer. At least 10,000 events were acquired. Analyses were performed using FlowJo flow cytometry anaysis software.

Chromatin immunoprecipitation. Cells were fixed with $1 \%$ formaldehyde for $15 \mathrm{~min}$ at room temperature. Fixation was stopped by adding glycine (to $0.125 \mathrm{M}$ ) with an additional incubation of $5 \mathrm{~min}$. Cells were collected by scraping, pelleted and then lysed for $10 \mathrm{~min}$ in $1 \mathrm{ml}$ of buffer LB1 (50 mM HEPES ( $\mathrm{pH}=7.5)$, $140 \mathrm{mM} \mathrm{NaCl}, 1 \mathrm{mM}$ EDTA, $0.5 \% \mathrm{NP}-40,0.25 \%$ Triton X-100 and 10\% glycerol) supplemented with protease inhibitors (Qiagen, Valencia, CA, USA). After centrifugation at $3,000 \mathrm{~g}$, pelleted nuclei were resuspended in $1 \mathrm{ml}$ of buffer LB2 $(10 \mathrm{mM}$ Tris $(\mathrm{pH}=8.0), 200 \mathrm{mM} \mathrm{NaCl}, 0.5 \mathrm{mM}$ EGTA and $1 \mathrm{mM}$ EDTA), and incubated at room temperature for $10 \mathrm{~min}$. Pelleted nuclei were resuspended in $1 \mathrm{ml}$ of ChIP SDS buffer (100 mM NaCl, $50 \mathrm{mM}$ Tris (pH 8), $5 \mathrm{mM}$ EDTA pH 8, $0.2 \% \mathrm{NaN}_{3}$ and $0.5 \% \mathrm{SDS}$ ) and sonicated for $20 \mathrm{~min}$ in a Covaris sonicator, yelding DNA fragments of 300-500 bp. Beads were blocked overnight in PBS with $0.5 \%$ BSA and then added to the samples. After a 3-h incubation at $4{ }^{\circ} \mathrm{C}$, beads were washed with Triton dilution buffer $(100 \mathrm{mM}$ Tris $(\mathrm{pH}=8.6), 100 \mathrm{mM} \mathrm{NaCl}, 5 \mathrm{mM}$ EDTA $(\mathrm{pH}=8), 0.2 \% \mathrm{NaN}_{3}$ and $5 \%$ Triton X-100), mixed micelle wash buffer ( $150 \mathrm{mM} \mathrm{NaCl}, 20 \mathrm{mM}$ Tris (pH 8), $5 \mathrm{mM}$ EDTA (pH 8), $5 \%$ sucrose, $0.2 \% \mathrm{NaN}_{3}$, $1 \%$ Triton X-100 and $0.2 \%$ SDS), Buffer 500 (0.1\% deoxycholic acid, $1 \mathrm{mM}$ EDTA $(\mathrm{pH}=8), 50 \mathrm{mM}$ HEPES $(\mathrm{pH}=7.5), 1 \%$ Triton $\mathrm{X}-100,500 \mathrm{mM} \mathrm{NaCl}$ and $0.2 \%$ $\mathrm{NaN}_{3}$ ), LiCl buffer (0.5\% deoxycholic acid, $1 \mathrm{mM} \mathrm{EDTA}(\mathrm{pH} 8), 250 \mathrm{mM} \mathrm{LiCl}$, $0.5 \%$ NP- $40,10 \mathrm{mM}$ Tris $(\mathrm{pH}=8)$ and $\left.0.2 \% \mathrm{NaN}_{3}\right)$ and Tris-EDTA (TE). DNA was eluted in elution buffer and crosslinks were reversed by incubation overnight at $65^{\circ} \mathrm{C}$. RNA and protein were digested using RNase A and Proteinase K and DNA was purified by phenol-chloroform extraction and isopropanol precipitation. Target DNA abundance in ChIP eluates was assayed by qPCR with primer pairs designed to achieve products of 50-200 bp. Primer sequences are provided in Supplementary Data 3 . The following antibodies were used: anti-MYC N262 (sc-764, Santa Cruz Biotechnology), anti-H3K4me3 (ab8580, Abcam), anti-panAc Histone H3 (06-599, Merk Millipore) and anti-Histone H3 (ab1791, Abcam).

DNase I hypersensitivity assay. Chromatin samples were subjected to DNAse I digestion. Chromatin $(2 \mu \mathrm{g})$ was treated with $0.5,1$ and 2 units of RQ1 RNase-Free DNAse I (Promega, Fitchburg, WI, USA) for $3 \mathrm{~min}$ at $37^{\circ} \mathrm{C}$ in $1 \times$ DNAse incubation buffer. Reactions were terminated by adding $2 \mathrm{mM}$ EGTA and the crosslinking was reversed by incubating samples at $65^{\circ} \mathrm{C}$. After $6 \mathrm{~h}$, proteinase $\mathrm{K}$ $\left(40 \mathrm{mg} \mathrm{ml}^{-1}\right)$ was added to each reaction and incubated overnight at $37^{\circ} \mathrm{C}$. After phenol-chloroform extraction, DNA was quantified and used as template for $\mathrm{qPCR}$ reactions with the same primer pairs used for ChIP-qPCR.

ChIP-Seq libraries and massive parallel sequencing. ChIP was performed as described above. DNA $(20 \mathrm{ng})$ was quantified by fluorimetry, resolved by electrophoresis and fractions of 50-250 bp were extracted. Input samples correspond to balanced blends of inputs from selected samples. Fractions were processed through subsequent enzymatic treatments of end repair, dA tailing and ligation to adaptors following Illumina's 'TruSeq DNA Sample Preparation Guide' (part \#15005180 Rev. C). Adaptor-ligated libraries were amplified by limited-cycle PCR with Illumina PE primers (12 cycles). The resulting purified DNA library was applied to an Illumina flow cell for cluster generation (TruSeq cluster generation kit v5) and sequenced on the Genome Analyzer IIx with SBS TruSeq v5 reagents following the manufacturer's protocols.

ChIP-Seq data processing. Image analysis and per-cycle base calling was performed with Illumina Real Time Analysis software (RTA1.13). Conversion to FASTQ read format with the ELAND algorithm (v2e) was performed with CASAVA-1.8 (Illumina). Quality check was done via fastqc (v0.9.4, Babraham Bioinformatics). ChIP-Seq reads were aligned to the human reference genome (GRCh37/hg19, February 2009) with Burrows-Wheeler Aligner (v0.5.9-r16) allowing 0-1 mismatches. Unique aligned reads were converted to BED format (Supplementary Data 4). All ChIP and input samples were normalized randomly to the same number of reads $(10,512.988)$. Furthermore, reads were directionally extended to $300 \mathrm{bp}$ and, for each base pair in the genome, the number of overlapping sequence reads was determined and averaged over a 10-bp window to create a wig file to visualize the data in the University of California Santa Cruz genome browser. The number of significant peaks of MYC-binding sites was 1,762 for $\mathrm{sh} \# 1+\mathrm{OHT}$ and 1,397 for shNt + OHT, using MACS (version

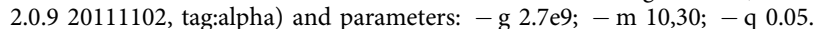

Motif-enrichment analysis. Motifs for the list of peaks in shNt + OHT were identified with the MEME suite and then TOMTOM was used to compare the identified motifs with known transcription factor-binding motifs. Sequence logos were generated using WebLogo 2.8.2.

Peak annotation and density plot analysis. Genomic annotation was carried out with Hypergeometric Optimization of Motif EnRichment (software v4.2). The tool annotatePeaks.pl was used with parameters by default and defined in the help. A gtf file from University of California Santa Cruz based on GRCh37/hg19 was used for annotations; the latter included whether a segment is in the TSS, transcription termination site, exon, $5^{\prime}$-untranslated region (UTR) exon, $3^{\prime}$-UTR exon, intron or is intergenic. Since some annotations overlap, the following priority was assigned: TSS (from $-1 \mathrm{~kb}$ to $+100 \mathrm{bp}$ ), transcription termination site (from $-100 \mathrm{bp}$ to $+1 \mathrm{~kb}$ ), protein coding exon, $5^{\prime}$-UTR exon, $3^{\prime}$-UTR exon, intron and intergenic. More detailed information is available in http://homer.salk.edu/homer/ngs/ annotation.html. The SeqMINER (v1.3.3e) platform ${ }^{73}$ was used to generate the density read plots shown in Supplementary Fig. 3a.

Gene Set Enrichment Analysis. MYC-bound genes were rank-ordered according to the fold change in FPKM (fragments per kilobase of exon per million fragments mapped) values (4-OHT versus vehicle) in HFF MYC-ER control cells and then submitted to analysis using GSEA software (www.broadinstitute.org/gsea). The list of pre-ranked genes was analysed with the gene set matrix composed file c2.all.v4.0.symbols.gmt and c5.all.v4.0.symbols.gmt. Significant gene sets enriched by 4-OHT treatment of control cells were identified using a false discovery rate q-value $<0.25$ and a nominal $P$ value $<0.05$, as defined by http://www.broadinstitute.org/gsea/doc/ GSEAUserGuideFrame.html?Interpreting_GSEA.

RNA-seq. Total RNA $(1 \mu \mathrm{g})$ was spiked with ERCC ExFold RNA spike-In mixes (Life Technologies). RNA quality was assessed on an Agilent 2100 Bioanalyzer and samples with a RNA integrity number $>8.5$ were used. PolyA + fractions were purified, randomly fragmented, converted to double-stranded cDNA and processed through subsequent enzymatic treatments of end repair, $\mathrm{dA}$ tailing and ligation to adaptors following Illumina 'TruSeq Stranded mRNA Sample Preparation Part \# 15031047 Rev. D' (this kit incorporates dUTP during second-strand cDNA synthesis, which implies that only the cDNA strand generated during first-strand synthesis is eventually sequenced). Adaptor-ligated libraries were generated by PCR with Illumina PE primers (eight cycles). The resulting purified cDNA libraries were applied to an Illumina flow cell for cluster generation (TruSeq cluster generation kit v5) and sequenced on the Genome Analyzer IIx with SBS TruSeq v5 reagents by following the manufacturer's protocols.

RNA-Seq data processing. Image analysis and per-cycle base-calling was performed with Illumina Real Time Analysis software (RTA1.13). Conversion to FASTQ read format with the ELAND algorithm (v2e) was performed with CASAVA-1.8 (Illumina). These files contain only reads that passed 'chastity' filtering (flagged with a ' $\mathrm{N}$ ', for 'not filtered' in the sequence identifier line). 'Chastity' parameter measures signal contamination in raw data and allows to flag unreliable reads. Quality check was done via fastqc (v0.9.4, Babraham Bioinformatics). Raw reads were aligned to the build version GRCh37/hg19 of the human genome where the sequence of the ERCC synthetic spike-in RNAs (http://tools.invitrogen.com/downloads/ERCC92.fa) had been added. Tophat5 (version 2.0.4) was used for alignment with the following parameters: --bowtiel, --max-multihits 5, --genome-read-mismatches 1 --segment-mismatches 1 --segment-length 19 --splice-mismatches 0 --library-type fr-firststrand. Gene expression levels and synthetic spike-in RNA (FPKM) were quantified with cufflinks (version 2.0.2), with the following parameters: -N, --library-type fr-firststrand, -u. Further, we used a loess regression to renormalize the FPKM values by using only the spike-in values to fit the loess following the strategy described ${ }^{74}$. The affy package in $\mathrm{R}$ provides a function, loess.normalize, performing loess regression on a matrix of values and allowing to specify which subset of data to use when fitting the loess (see the affy package for further details). The result was a matrix of FPKM values normalized to the control ERCC spike-ins.

RNA-Seq GSEA analysis. Genes were rank-ordered according to the fold change in FPKM values (4-OHT versus vehicle) in HFF MYC-ER control cells and then submitted to analysis using GSEA software (www.broadinstitute.org/gsea). The list of pre-ranked genes was analysed with the gene set matrix composed file c2.all.v4.0.symbols.gmt and c5.all.v4.0.symbols.gmt. Significant gene sets enriched by OHT treatment of control cells were identified using a false discovery rate q-value $<0.25$ and a nominal $P$ value $<0.05$. All analyses were performed using GSEA v2.1 software with pre-ranked list and 1000 data permutations. 
Analysis of human genomic tumour data. Gene expression data from 20 studies profiling human tumours were downloaded from either Oncomine or GEO. The complete list of data sets, together with their GEO accession numbers, is provided in Supplementary Data 9. Expression data for each study were converted into the GenePattern GCT format. To obtain one expression value per gene and sample, GCT files were subsequently collapsed using the CollapseDataset module in GenePattern. We next rank-ordered the samples within each data set according to the mRNA levels of BPTF, c-MYC, N-MYC or L-MYC and performed a ssGSEA to calculate activation scores for four MYC-dependent gene signatures in each sample. ssGSEA-enrichment score represents the degree to which the genes in a particular gene set are coordinately up- or downregulated within a sample. The following gene signatures were downloaded from Molecular Signature Database: BILD_MYC_ONCOGENIC_SIGNATURE (M2069), ALFANO_MYC_TARGETS (M2477) and SCHUHMACHER_MYC_TARGETS_UP. The Seitz signature was built from the data published in ref. 75 .

Statistical analysis. All quantitative data are presented as mean \pm s.e.m. from $\geq 3$ experiments or samples per data point ( $n$ is mentioned in each figure legend). Unpaired Student's $t$-test (two-tailed) was used to compare two groups of independent samples. Paired Student's $t$-test (two-tailed) was used to compare matched pairs samples. To compare the data distribution of two separate populations without assuming normal distribution, we performed a Wilcoxon signed-ranked test. For in vitro experiments, sample size required was not determined a priori. The experiments were not randomized.

\section{References}

1. Vita, M. \& Henriksson, M. The Myc oncoprotein as a therapeutic target for human cancer. Semin. Cancer Biol. 16, 318-330 (2006).

2. Tansey, W. P. Mammalian MYC proteins and cancer. New J. Sci. 2014, 1-27 (2014).

3. Blackwood, E. M. \& Eisenman, R. N. Max: a helix-loop-helix zipper protein that forms a sequence-specific DNA-binding complex with Myc. Science 251, 1211-1217 (1991).

4. Blackwell, T. K. et al. Binding of Myc proteins to canonical and noncanonical DNA sequences. Mol. Cell Biol. 13, 5216-5224 (1993).

5. Fernandez, P. C. et al. Genomic targets of the human c-Myc protein. Genes Dev. 17, 1115-1129 (2003).

6. Guccione, E. et al. Myc-binding-site recognition in the human genome is determined by chromatin context. Nat. Cell Biol. 8, 764-770 (2006).

7. Sabò, A. et al. Selective transcriptional regulation by Myc in cellular growth control and lymphomagenesis. Nature 511, 488-492 (2014).

8. Cheng, S. W. et al. c-MYC interacts with INI1/hSNF5 and requires the SWI/SNF complex for transactivation function. Nat. Genet. 22, 102-105 (1999).

9. Rahl, P. B. et al. c-Myc regulates transcriptional pause release. Cell 141, 432-445 (2010).

10. Thomas, L. R. et al. Interaction with WDR5 promotes target gene recognition and tumorigenesis by MYC. Mol. Cell 58, 440-452 (2015).

11. Langst, G. et al. Nucleosome movement by CHRAC and ISWI without disruption or trans-displacement of the histone octamer. Cell 97, 843-852 (1999).

12. Xiao, H. et al. Dual functions of largest NURF subunit NURF301 in nucleosome sliding and transcription factor interactions. Mol. Cell 8, 531-543 (2001).

13. Vicent, G. P. et al. Four enzymes cooperate to displace histone H1 during the first minute of hormonal gene activation. Genes Dev. 25, 845-862 (2011).

14. Alkhatib, S. G. \& Landry, J. W. The nucleosome remodeling factor. FEBS Lett. 585, 3197-3207 (2011).

15. Balbás-Martínez, C. et al. Recurrent inactivation of STAG2 in bladder cancer is not associated with aneuploidy. Nat. Genet. 45, 1464-1469 (2013).

16. Landry, J. et al. Essential role of chromatin remodeling protein Bptf in early mouse embryos and embryonic stem cells. PLoS Genet. 4, e1000241 (2008).

17. Neri, F. et al. Myc regulates the transcription of the PRC2 gene to control the expression of developmental genes in embryonic stem cells. Mol. Cell. Biol. 32, 840-851 (2012).

18. Lovén, J. et al. Revisiting global gene expression analysis. Cell 151, 476-482 (2012).

19. Schuhmacher, M. et al. The transcriptional program of a human B cell line in response to Myc. Nucleic Acids Res. 29, 397-406 (2001).

20. Schlosser, I. et al. Dissection of transcriptional programmes in response to serum and c-Myc in a human B-cell line. Oncogene 24, 520-524 (2005).

21. Acosta, J. C. et al. Myc inhibits p27-induced erythroid differentiation of leukemia cells by repressing erythroid master genes without reversing p27mediated cell cycle arrest. Mol. Cell. Biol. 28, 7286-7295 (2008).

22. Kim, Y. H. et al. Combined microarray analysis of small cell lung cancer reveals altered apoptotic balance and distinct expression signatures of MYC family gene amplification. Oncogene 25, 130-138 (2006).
23. O'Donnell et al. Activation of transferrin receptor 1 by c-Myc enhances cellular proliferation and tumorigenesis. Mol. Cell. Biol. 26, 2373-2386 (2006).

24. Frank, S. R. et al. MYC recruits the TIP60 histone acetyltransferase complex to chromatin. EMBO Rep. 4, 575-580 (2003).

25. Nagy, Z. \& Tora, L. Distinct GCN5/PCAF-containing complexes function as co-activators and are involved in transcription factor and global histone acetylation. Oncogene 26, 5341-5357 (2007).

26. Perna, D. et al. Genome-wide mapping of Myc binding and gene regulation in serum-stimulated fibroblast. Oncogene 31, 1695-1709 (2012).

27. Bailey, T. L. et al. MEME SUITE: tools for motif discovery and searching. Nucleic Acids Res. 37, W202-W208 (2009).

28. Di Stefano, B. et al. C/EBP $\alpha$ poises B cells for rapid reprogramming into induced pluripotent stem cells. Nature 506, 235-239 (2014).

29. Thurman, R. E. et al. The accessible chromatin landscape of the human genome. Nature 489, 75-82 (2012).

30. Liu, Y. C. et al. Global regulation of nucleotide biosynthetic genes by c-Myc. PLoS ONE 3, e2722 (2008).

31. Dominguez-Sola, D. et al. Non-transcriptional control of DNA replication by c-Myc. Nature 448, 445-451 (2007).

32. Murga, M. et al. Exploiting oncogene-induced replicative stress for the selective killing of Myc-driven tumors. Nat. Struct. Biol. 18, 1331-1335 (2011).

33. Evan, G. I. et al. Induction of apoptosis in fibroblasts by c-myc protein. Cell 69, 119-128 (1992).

34. Taub, R. et al. Translocation of the c-myc gene into the immunoglobulin heavy chain locus in human Burkitt lymphoma and murine plasmacytoma cells. Proc. Natl Acad. Sci. USA 79, 7837-7841 (1982).

35. Sansom, O. J. et al. Myc deletion rescues Apc deficiency in the small intestine. Nature 446, 676-679 (2007)

36. Taylor, B. S. et al. Integrative genomic profiling of human prostate cancer. Cancer Cell 18, 11-22 (2010).

37. Garson, J. A. et al. N-myc gene expression and oncoprotein characterization in medulloblastoma. Br. I. Cancer 59, 889-894 (1989).

38. Wu, R. et al. Amplification and overexpression of the L-MYC proto-oncogene in ovarian carcinomas. Am. J. Pathol. 162, 1603-1610 (2003).

39. Barbie, D. A. et al. Systematic RNA interference reveals that oncogenic KRAS-driven cancers require TBK1. Nature 462, 108-112 (2009).

40. Martinelli, P. et al. Gata6 is required for complete acinar differentiation and maintenance of the exocrine pancreas in adult mice. Gut 62, 1481-1488 (2013).

41. Flandez, M. et al. Nr5a2 heterozygosity sensitises to, and cooperates with, inflammation in KRas(G12V)-driven pancreatic tumourigenesis. Gut 63, 647-655 (2014).

42. Sandgren, E. P. et al. Pancreatic tumor pathogenesis reflects the causative genetic lesion. Proc. Natl Acad. Sci. USA 88, 93-97 (1991).

43. Aguilar, S. et al. Tissue plasminogen activator in murine exocrine pancreas cancer: selective expression in ductal tumours and contribution to cancer progression. Am. J. Pathol. 165, 1129-1139 (2004).

44. Kress, T. R. et al. MYC: connecting selective transcriptional control to global RNA production. Nat. Rev. Cancer. 15, 593-607 (2015).

45. Wysocka, J. et al. A PHD finger of NURF couples histone H3 lysine 4 trimethylation with chromatin remodelling. Nature 442, 86-90 (2006).

46. Martinato, F. et al. Analysis of Myc induced histone modifications on target chromatin. PloS One 3, e3650 (2008).

47. Carré, C. et al. The Drosophila NURF remodelling and the ATAC histone acetylase complexes functionally interact and are required for global chromosome organization. EMBO Rep. 9, 187-192 (2008).

48. Hill, D. A. \& Imbalzano, A. N. Human SWI/SNF nucleosome remodeling activity is partially inhibited by linker histone H1. Biochemistry 39, 11649-11656 (2000).

49. Qiu, Z. et al. Functional interactions between NURF and Ctcf regulate gene expression. Mol. Cell. Biol. 35, 224-237 (2015).

50. Heintzman, N. D. et al. Distinct and predictive chromatin signatures of transcriptional promoters and enhancers in the human genome. Nat. Genet. 39, 311-318 (2007).

51. Krebs, A. R. et al. SAGA and ATAC histone acetyl transferase complexes regulate distinct sets of genes and ATAC defines a class of p300-independent enhancers. Mol. Cell 44, 410-423 (2011).

52. Smallwood, A. \& Ren, B. Genome organization and long-range regulation of gene expression by enhancers. Curr. Opin. Cell Biol. 25, 387-394 (2013).

53. Ruthenburg, A. J. et al. Recognition of a mononucleosomal histone modification pattern by BPTF via multivalent interactions. Cell 145, 692-706 (2011).

54. Schnetz, M. P. et al. CHD7 targets active gene enhancer elements to modulate ES cell-specific gene ex-pression. PLoS Genet. 6, pe1001023 (2010).

55. Rada-Iglesias, A. et al. A unique chromatin signature uncovers early developmental enhancers in humans. Nature 470, 279-283 (2011).

56. Soucek, L. et al. Omomyc, a potential Myc dominant negative, enhances Mycinduced apoptosis. Cancer Res. 62, 3507-3510 (2002). 
57. Savino, M. et al. The action mechanism of the Myc inhibitor termed Omomyc may give clues on how to target Myc for cancer therapy. PLoS One 6, e22284 (2011).

58. Hoffman, B. \& Liebermann, D. A. Apoptotic signaling by c-MYC. Oncogene 27, 6462-6472 (2008).

59. Nesbit, C. E., Tersak, J. M. \& Prochownik, E. V. MYC oncogenes and human neoplastic disease. Oncogene 18, 3004-3016 (1999).

60. Schwab, M., Varmus, H. E. \& Bishop, J. M. Human N-myc gene contributes to neoplastic transformation of mammalian cells in culture. Nature 316, 160-162 (1985).

61. Malynn, B. A. et al. N-myc can functionally replace c-myc in murine development, cellular growth, and differentiation. Genes Dev. 14, 1390-1399 (2000).

62. Stanton, B. R. et al. Loss of N-myc function results in embryonic lethality and failure of the epi- thelial component of the embryo to develop. Genes Dev. 6, 2235-2247 (1992).

63. Gombert, W. M. \& Krumm, A. Targeted deletion of multiple CTCF-binding elements in the human C-MYC gene reveals a requirement for CTCF in C-MYC expression. PLoS One 4, e6109 (2009).

64. Prochownik, E. V. \& Vogt, P. K. Therapeutic targeting of Myc. Genes Cancer 1, 650-659 (2010).

65. Arvanitis, C. \& Felsher, D. W. Conditional transgenic models define how MYC initiates and maintains tumorigenesis. Semin. Cancer Biol. 16, 313-317 (2006).

66. Waddell, N. et al. Whole genomes redefine the mutational landscape of pancreatic cancer. Nature 518, 495-501 (2015).

67. Saborowski, M. et al. A modular and flexible ESC-based mouse model of pancreatic cancer. Genes Dev. 28, 85-97 (2014).

68. Dawson, M. et al. Inhibition of BET recruitment to chromatin as an effective treatment for MLL-fusion leukaemia. Nature 478, 529-533 (2011).

69. Delmore, J. E. et al. BET bromodomain inhibition as a therapeutic strategy to target c-Myc. Cell 146, 904-917 (2011).

70. Jones, M. H., Hamana, N. \& Shimane, M. Identification and characterization of BPTF, a novel bromodomain transcription factor. Genomics 63, 35-39 (2000).

71. Kawaguchi, Y. et al. The role of the transcriptional regulator Ptfla in converting intestinal to pancreatic progenitors. Nat Genet. 32, 128-34 (2002)

72. Burlison, J. S. et al. Pdx-1 and Ptfla concurrently determine fate specification of pancreatic multipotent progenitor cells. Dev. Biol. 316, 74-86 (2008).

73. Ye, T. et al. seqMINER: an integrated ChIP-seq data interpretation platform. Nucleic. Acids. Res. 39, e35 (2010).

74. Lovén, J. et al. Revisiting global gene expression analysis. Cell 151, 476-82 (2012).

75. Seitz, V. et al. Deep sequencing of MYC DNA-binding sites in Burkitt lymphoma. PLoS One 6, e26837 (2011)

\section{Acknowledgements}

We thank the members of the Epithelial Carcinogenesis Group for valuable discussions; N. del Pozo for valuable contributions with animal experimentation; A. Picornell for valuable contributions in an early phase of the project; $\mathrm{M}$. Soengas for providing cells; M. Magnuson and C. V. Wright for providing mice; B. Amati, M. Beato, O. Fernández Capetillo, M. Serrano, G. Vicent and A. Sabò for providing critical comments to prior versions of the manuscript. This work was supported, in part, by grants from Ministerio de Economía y Competitividad, Madrid, Spain (SAF2007-60860, SAF2011-29530 and ONCOBIO Consolider), Instituto de Salud Carlos III, Madrid, Spain (RTICC RD12/0036/0034), European Union Seventh Framework Programme (grant 256974), and an EIN/Roche-CNIO collaborative grant to F.X.R.; and grants from Instituto de Salud Carlos III (INTRASALUD PI12/00425 and RTICC RD12/0036/0037) to J.C.C.

\section{Author contributions}

L.R. designed and performed the majority of the experiments and M.P.d.A. assisted with some of the experiments. A.R.-M. designed and performed experiments with BL cells. E.C.-d.S.P. was responsible for bioinformatics analysis of the data. All authors contributed in experimental design and data analysis. V.J.S.-A.L. and F.X.R. designed and supervised the overall conduct of the study. L.R., V.J.S.-A.L., and F.X.R. wrote the paper with contributions from the other coauthors. F.X.R. and J.C.C. obtained financial support.

\section{Additional information}

Accession codes: ChIP-Seq and RNA-Seq Data sets have been deposited at GEO with the following accession numbers GSE65544 (ChIP-Seq), GSE65545 (RNA-Seq) and GSE65546 (the combined data set).

Supplementary Information accompanies this paper at http://www.nature.com/ naturecommunications

Competing financial interests: The authors declare no competing financial interests.

Reprints and permission information is available online at http://npg.nature.com/ reprintsandpermissions/

How to cite this article: Richart, L. et al. Bptf is required for c-MYC transcriptional activity and in vivo tumorigenesis. Nat. Commun. 7:10153 doi: 10.1038/ncomms10153 (2016).

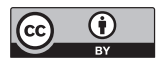

This work is licensed under a Creative Commons Attribution 4.0 International License. The images or other third party material in this article are included in the article's Creative Commons license, unless indicated otherwise in the credit line; if the material is not included under the Creative Commons license, users will need to obtain permission from the license holder to reproduce the material. To view a copy of this license, visit http://creativecommons.org/licenses/by/4.0/ 